\title{
Antidepressant-like effects of the Guanxin Danshen formula via mediation of the CaMK II-CREB-BDNF signalling pathway in chronic unpredictable mild stress-induced depressive rats
}

\author{
Weijie Xie ${ }^{1,2,3,4}$, Xiangbao Meng ${ }^{1,2,3,4}$, Yadong Zhai ${ }^{1,2,3,4}$, Tianyuan Ye ${ }^{1,2,3,4}$, Ping Zhou ${ }^{1,2,3,4}$, Fengwei Nan ${ }^{1,2,3,4}$, \\ Guibo Sun ${ }^{1,2,3,4}$, Xiaobo Sun ${ }^{1,2,3,4}$
}

${ }^{1}$ Beijing Key Laboratory of Innovative Drug Discovery of Traditional Chinese Medicine (Natural Medicine) and Translational Medicine, Institute of Medicinal Plant Development, Peking Union Medical College and Chinese Academy of Medical Sciences, Beijing 100193 , China; ${ }^{2}$ Key Laboratory of Bioactive Substances and Resource Utilization of Chinese Herbal Medicine, Ministry of Education, Beijing 100193, China; ${ }^{3}$ Key Laboratory of Efficacy Evaluation of Chinese Medicine against Glycolipid Metabolic Disorders, State Administration of Traditional Chinese Medicine, Beijing 100193, China; ${ }^{4}$ Zhongguancun Open Laboratory of the Research and Development of Natural Medicine and Health Products, Beijing 100193, China

Contributions: (I) Conception and design: W Xie, Y Zhai, T Ye; (II) Administrative support: G Sun, X Sun; (III) Provision of study materials or patients: G Sun, X Sun; (IV) Collection and assembly of data: W Xie, X Meng, F Nan; (V) Data analysis and interpretation: W Xie, P Zhou; (VI) Manuscript writing: All authors; (VII) Final approval of the manuscript: All authors.

Correspondence to: Guibo Sun; Xiaobo Sun. Beijing Key Laboratory of Innovative Drug Discovery of Traditional Chinese Medicine (Natural Medicine) and Translational Medicine, Institute of Medicinal Plant Development, Peking Union Medical College and Chinese Academy of Medical Sciences, Beijing 100193, China. Email: sunguibo@126.com; sun_xiaobo163@163.com.

Background: Depression is a chronic and recurrent syndrome of mood disorder causing immense social and economic burden; thus, treatment should be improved. Guanxin Danshen formula (GXDSF), a natural botanical drug composition prescription, has significant cardiovascular protective effects and is widely used in the clinical treatment of myocardial ischaemic diseases. However, it is still unclear and seldom studied whether GXDSF has neuroprotective effects against depressive disorders. This study explored whether GXDSF has antidepressant-like effects in rats exposed to chronic unpredictable mild stress (CUMS) and analysed the possible underlying neurotrophic expression and psychotropic mechanisms.

Methods: The present study was designed to investigate the antidepressant effects of GXDSF treatment in a CUMS-induced rat model. Based on the clinical doses, the drug-treated group was intragastrically administered GXDSF for 30 days, and rats were simultaneously exposed to CUMS stimulation for 30 days. After induction and drug administration, the depression-like behaviours were determined via the sucrose preference test (SPT), the open field test (OFT), the tail suspension test (TST), and the forced swim test (FST). ELISA kits were used to examine the monoaminergic neurotransmitters, monoamine oxidase (MAO) and $\mathrm{Ca}^{2+}$ levels in the hippocampus. Moreover, we measured and analysed the brain-derived neurotrophic factor (BDNF) and nerve growth factor (NGF) levels and the upstream regulation and signal pathways of BDNF and NGF to explore their related mechanisms in this animal model of depression, including calciumcalmodulin dependent protein kinase-II (CaMKII) and cAMP response element-binding (CREB).

Results: The results revealed that GXDSF may possess significant antidepressant-like effects via improving body weight, raising the sucrose preference in the SPT, increasing the total distance, the number of upright stands, and the residence time of the central zone in the open field test (OPF) and reducing the immobility time in the TST and FST. In addition, GXDSF significantly upregulated the relative levels of neurotransmitters, including dopamine (DA), norepinephrine (NE), and serotonin (5HT), in a dose-dependent manner and inhibited MAO activities in the hippocampus. Moreover, GXDSF reversed the decline in intracellular CREB and p-CREB expression induced by CUMS, downregulated the phosphorylation levels of intracellular CaMKII and its two subunits CaMKII $\alpha$ and CaMKII $\beta$ in the hippocampus, and thus, clearly upregulated the downstream effector protein expression levels of BDNF, 
NGF, and synitaxine-1 in the hippocampus. These data suggest that the antidepressant effects of GXDSF have a potential relationship with regulating changes in the CaMKII-CREB-BDNF pathway.

Conclusions: Despite several limitations of this study, the results have suggested that GXDSF administration possesses antidepressant-like effects in CUMS-treated rats and provide the first in vivo demonstration of a possible mechanism of GXDSF via regulating changes in the CaMKII-CREB-BDNF signalling pathway. These findings provide a novel potential substrate by which herbal antidepressants may exert therapeutic effects in the treatment of depression.

Keywords: Guanxin Danshen formula (GXDSF); chronic unpredictable mild stress (CUMS); antidepressant; neurotransmitters; calcium-calmodulin dependent protein kinase-II (CaMKII); brain-derived neurotrophic factor (BDNF)

Submitted Jun 21, 2019. Accepted for publication Aug 21, 2019.

doi: 10.21037/atm.2019.09.39

View this article at: http://dx.doi.org/10.21037/atm.2019.09.39

\section{Introduction}

Depression is a chronic and recurrent syndrome of mood disorders that is closely associated with stress exposure and affects $17 \%$ to $20 \%$ of the population worldwide (1). According to the World Health Organization, depression will rank as the second leading cause of disability worldwide by 2020, resulting in a major social and economic burden (2). The main symptoms of depression are fatigue, depressed mood, loss of pleasure, social isolation and loss of energy; depression is also associated with cognitive dysfunction, loss of appetite, fatigue, sleep disorders and other endocrine or metabolic alterations (3). Current drugs used clinically, including selective serotonin reuptake inhibitors (SSRIs), tricyclic antidepressants (TCAs), monoamine oxidase inhibitors (MAOIs), noradrenergic reuptake inhibitors (NARIs), serotonin and noradrenaline reuptake inhibitors (SNRIs), exhibit a low curative ratio and various side effects (4). Thus, there is an urgent need to explore and develop new antidepressants with higher efficacy and fewer side effects.

Current evidence suggests that the occurrence of depression may be related to a reduced secretion of neurotransmitters (5-7), such as dopamine (DA), norepinephrine (NE), and serotonin (5-HT), neuronal apoptosis (8-10), inflammation (11-14), intestinal flora (9) and other factors $(6,9,15,16)$. It has been proven that antidepressant and anti-anxiety effects may occur by increasing the levels of the brain monoamine neurotransmitters 5-HT $(17,18)$ and $\mathrm{NE}$ in the central nervous system. It is also widely acknowledged that the brain-derived neurotrophic factor (BDNF) and its mediated signalling pathways (its cognate receptor TrkB) may participate in many behavioural and molecular mechanisms of antidepressants via alterations of synaptic plasticity, neuronal circuit formation, and neuronal survival (3,19-22).

Experiments have shown that the saponins of Panax Notoginseng (PNS) and P. ginseng (GTS) promote the release of neurotransmitters $5-\mathrm{HT}, \mathrm{NE}$, and DA, modulate the secretion of $\mathrm{Ca}^{2+}$ concentrations, and increase nestin and $\operatorname{BDNF}(8,10,23-26)$; additionally, PNS regulates the calmodulin kinase channel (CaMK) and multiple upstream neural signal transduction pathways. Therefore, PNS and GTS may exert their antidepressant-like effects. The Guanxin Danshen formula (GXDSF), a traditional Chinese herbal composition prescription, has been recorded in the Chinese Pharmacopeia since the 1995 edition $(27,28)$. It consists of Salviae miltiorrhizae Radix, Notoginseng Radix (PNS) and Dalbergiae odoriferae Lignum, meaning that the GXDSF active ingredients are PNS, tanshinone and volatile oil compounds $(27,29,30)$. Hence, based on the fact that GXDSF contains PNS, it can be speculated that GXDSF may possess neuroprotective effects against depression.

Previous research reports have indicated that GXDSF had significant cardiovascular protective effects and has been widely used in the clinical treatment of myocardial ischaemic diseases (27-31), including inhibition of the differentiation of myocardial fibroblasts and myocardial ischaemia reperfusion injury (MIRI), downregulation of myocardial infarct size, maintenance of myocardium structure, and improvement of cardiac function in rats with MIRI. However, there were no related reports on whether GXDSF had neuroprotective effects against depressive disorders.

Based on the effects of PNS, it was hypothesized that GXDSF could ameliorate depression-like behaviours 
Table 1 Drug composition and animal treatment doses of GXDSF. GXDSF stands for Guanxin Danshen formula; Volatile oil of DOL stands for volatile oil extract of Dalbergiae odoriferae Lignum

\begin{tabular}{|c|c|c|c|c|}
\hline Group & Formula & Drug Composition & Clinical dose $(70 \mathrm{~kg})$ & Rat treatment dose $(1 \mathrm{~kg})$ \\
\hline \multirow{2}{*}{ High-dose group $(\mathrm{H})$} & & Notoginseng Radix (g) & 7.20 & 0.648 \\
\hline & & Volatile oil of DOL $(\mu \mathrm{L})$ & 63.00 & 5.670 \\
\hline \multirow[t]{2}{*}{ Moderate-dose group (M) } & 2-fold GXDSF & Salviae miltiorrh Radix (g) & 3.60 & 0.324 \\
\hline & & Volatile oil of DOL $(\mu \mathrm{L})$ & 31.50 & 2.835 \\
\hline \multirow[t]{3}{*}{ Low-dose group (L) } & 1-fold GXDSF & Salviae miltiorrh Radix (g) & 1.80 & 0.162 \\
\hline & & Notoginseng Radix (g) & 1.80 & 0.162 \\
\hline & & Volatile oil of DOL $(\mu \mathrm{L})$ & 15.75 & 1.418 \\
\hline
\end{tabular}

induced by CUMS. The present study was designed to investigate the antidepressant effects of GXDSF treatment in the CUMS-induced model rats. After induction, the depression-like behaviours were determined via the sucrose preference test, the open field test, the tail suspension test, and the forced swim test. Moreover, we also examined the monoaminergic neurotransmitters, the BDNF expression levels, and the upstream regulation of BDNF to explore its related mechanism in this animal model of depression.

\section{Methods}

\section{Animals}

Male Sprague Dawley (SD) rats (SPF, weighing 280-300 g) were used in this study, and they were all purchased from Beijing Vital Lihua Experimental Animals Co., Ltd., whose license number was SCXK (Beijing) 2016-0006. The certificate number for the rats is 11400700289611 . Rats were housed at an ambient temperature of $20 \pm 1{ }^{\circ} \mathrm{C}$ and a relative humidity of $55 \% \pm 15 \%$ with artificial light for $12 \mathrm{~h}$ each day and free access to a standard laboratory chow diet and sterilized drinking water throughout the experiments. All efforts were made to minimize the number of animals used and their suffering. The study was conducted in accordance with the Declaration of Helsinki. The protocol was approved by the Laboratory Animal Ethics Committee of the Institute of Medicinal Plant Development, Peking Union Medical College, and conformed to the Guide for the Care and Use of Laboratory Animals (Permit Number: SYXK 2017-0020).

\section{Groups and drug administration}

As previously described in the base studies $(27,29)$, the Salviae miltiorrbizae Radix et Rhizoma, the Notoginseng Radix et Rhizoma and the oil extract of Dalbergiae odoriferae Lignum were mixed to form the natural botanical drug composition of GXDSF according to the Chinese Pharmacopoeia 2015. All of the medical raw materials for the GXDSF used in this study were provided by Zhongfa Industrial and Commercial Group, Yerui Pharmaceutical Co., Ltd. (Heilongjiang, China), and the batch number was NO. 20170501. The remaining medicinal materials for the GXDSF were obtained from the sample room of the Institute of Medicinal Plants, Chinese Academy of Medical Sciences (S20170512001). Moreover, the main chemical contents of the representative chemical components used in the GXDSF preparation were determined by HPLC, as shown in Figure S1.

As shown in Table 1, the low-dose group received the single-fold recommended clinical dose of GXDSF, the moderate-dose group received double the clinical dose, and the high-dose group received quadruple the clinical dose. The drug composition and animal-treated doses of GXDSF are shown in Table 1 and, based on the animal-treatment doses, the total formula powders were dissolved in normal saline with a $0.5 \%$ carboxymethylcellulose solution. Then, they were intragastrically administered daily in a constant volume.

Rats were divided into 6 groups (15 for each group), namely, the control group, the CUMS-induced model group, the low-dose group, the moderate-dose group, 


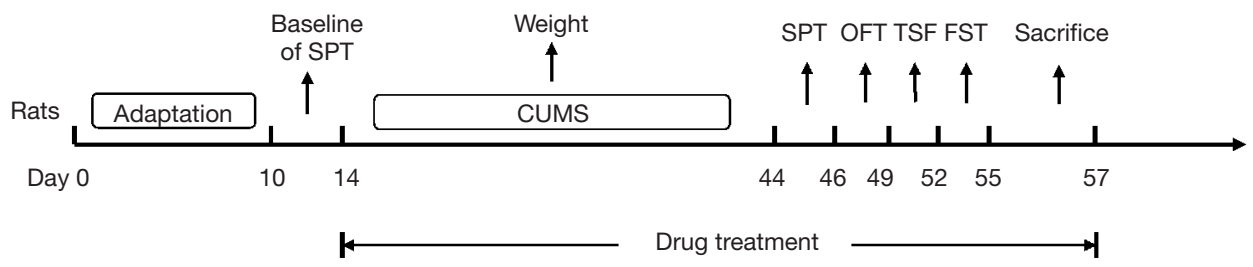

Figure 1 Experimental design and procedures of the CUMS-induced rat models. (I) Habituation for 10 days; (II) the baseline data of sucrose preference was determined on the 13th day; (III) 14-44th days, the CUMS induction and the intragastric administration of GXDSF; (IV) 44-46th days, sucrose preference test (SPT); (V) 46-49th days, open field test (OFT); (VI) 49-52nd days, tail suspension test (TST); (VII) 52-55th days, forced swim test (FST). On the 52-55th day, the animals were sacrificed after the behaviour tests, and blood and brain tissue were taken for analysis.

the high-dose group (Table 1), and the positive drug group at a dose of $2 \mathrm{mg} \cdot \mathrm{kg}^{-1}$ fluoxetine, according to the random number table. The study drugs were administered intragastrically for all animals in the drug-treated groups, and the animals in the control and model groups were given an equal volume of saline water. Continuous gastric administration (once per day) lasted for 30 days. During the behavioural tests, the drug administration continued, as shown in Figure 1.

\section{CUMS-induced rat model}

To explore the effects of GXDSF on depression-like behaviours in animals, the CUMS-induced rat model was used in this study. As shown in Figure 1, after habituation for 10 days, the sucrose preference test (SPT) was carried out. Rats were divided into six groups according to the baseline sucrose preference data on the 13 th day.

Then, the CUMS procedure was performed as previously described with minor modifications (19,32). Briefly, the control group was free of any of the chronic unpredictable mild stresses conducted by this experimental, whereas each rat in the CUMS-induced groups was single housed and subjected to one of the following stressors per day in a randomized fashion: (I) food and water deprivation for $12 \mathrm{hr}$, (II) light/dark perversion for $24 \mathrm{hr}$, (III) switching cages for $3 \mathrm{hr}$, (IV) soiled cage $(200 \mathrm{~mL}$ water in $100 \mathrm{~g}$ sawdust bedding) for $12 \mathrm{hr}$, (V) predator sounds for $30 \mathrm{~min}$ (cat), (VI) paired housing for $24 \mathrm{hr}$, (VII) cold swimming $\left(4^{\circ} \mathrm{C}, 5 \mathrm{~min}\right)$, (VIII) swimming in hot water $\left(40^{\circ} \mathrm{C}, 5 \mathrm{~min}\right.$ ), (IX) tail pinch ( $2 \mathrm{~cm}$ from the beginning of the tail) for $4 \mathrm{~min},(\mathrm{X})$ restraint stress for $4 \mathrm{hr}$, and (XI) soiled cage and foot shock (10 min). Rats were subjected to different stressors daily for 30 days in a chronic and unpredictable way.

\section{Behavioural tests in the CUMS model}

The experimental design procedure is shown in Figure 1. After habituation for 10 days, the sucrose preference test was carried out. The rats were then divided into six groups according to the baseline sucrose preference on the $13^{\text {th }}$ day. Following the CUMS induction period, all test groups were exposed to chronic stress for 30 days. Intragastric GXDSF was administered daily during the 30-day CUMS session and the behavioural test periods. On Day 44, behavioural tests were conducted as follows: SPT, OFT, TST, and FST. Animals were sacrificed after the behavioural tests; blood and brain tissues were taken for analysis.

\section{Weight and Sucrose preference test}

During the CUMS induction period, all rats in each group were weighed once per 7 days. In addition, on the 44-46th days, the SPT was performed before and after CUMS induction as previously reported with slight modification $(3,20,32)$. First, rats were housed in individual cages and each rat was adapted to a $1 \%$ sucrose solution $(\mathrm{w} / \mathrm{v}): 24 \mathrm{~h}$ exposure to two bottles of sucrose solution and an additional $24 \mathrm{~h}$ exposure to one bottle of $1 \%$ sucrose solution on the right side of each cage and one bottle of water on the left side. Then, all the rats were deprived of water and food for $12 \mathrm{hr}$, and the sucrose preference test was conducted for $1 \mathrm{~h}$. During this period, rats were given free access to two bottles, one containing 200 millilitres of $1 \%$ sucrose solution (right side) and the other 200 millilitres of water (left side). The position of the bottles was switched at the mid-point of testing to avoid possible side-preference effects. The sucrose preference was measured as a percentage of the consumed sucrose solution relative to the total amount of liquid intake. 


\section{Open field test}

After $30 \mathrm{~min}$, of the last intragastric administration, the open field test was performed to measure the locomotor activity of the animals on the 46-49th days $(3,32)$. To allow adaptation to the environment, all animals were taken to the test room $60 \mathrm{~min}$ before the test. The test time was from 8:30 to $16: 30$, and the room was dim and quiet during the experiment. The open field test device was purchased from Shanghai Xinsoft Information Technology Co., Ltd. (XR-XZ301, Shanghai, China); it consisted of square plastic pools $(100 \times 100 \mathrm{~cm}$ in the width and length, and $40 \mathrm{~cm}$ in height), a video camera and a light source of $60 \mathrm{~lx}$ hung over each. The open field box was divided into 25 equal squares and included three zones: outer squares, inner squares, and a centre grid. After each rat (all groups) was placed into the centre grid of the open field box, the square distance and the number of times the rat travelled through the squares in 5 min were recorded by the software tracking and analysis system (Shanghai Xinsoft Information Technology Co., Ltd, Shanghai, China). After that, the maze was wiped and cleaned with $75 \%$ alcohol to avoid affecting the test of the latter rats.

\section{Tail suspension test}

After 30 minutes of the last intragastric administration, the tail suspension test (TST) was performed to measure the immobility time of the rats on the 49-52nd days $(3,32)$. To allow adaptation to the environment, all animals were taken to the test room $60 \mathrm{~min}$ before the test. The test time was from 8:30 to $16: 30$, and the room was dim and quiet during the experiment. Briefly, rats were individually suspended upside down by the tail, $50 \mathrm{~cm}$ above the floor, using adhesive tape (approximately $1 \mathrm{~cm}$ from the tail tip). Each rat was suspended for $6 \mathrm{~min}$, and the procedure consisted of two sessions: an initial 2-min habituation followed by a 4-min recording session; during the last $4 \mathrm{~min}$, the immobility time was measured by the software tracking and analysis system (Shanghai Xinsoft Information Technology Co., Ltd, Shanghai, China). Rats were considered immobile only when they remained completely motionless. After each rat was tested, the maze was wiped and cleaned with $75 \%$ alcohol to avoid affecting the test of the latter rats.

\section{Forced swimming test}

After $30 \mathrm{~min}$ of the last intragastric administration, to measure the immobility time of the animals on the 52-55th day, the forced swimming test (FST) was undertaken as described previously with a modification $(3,32)$. To allow adaptation to the environment, all rats were taken to the test room for an initial 6 min pre-test followed $24 \mathrm{~h}$ later by a 6 min FST. The test time was from 8:30 to 16:30, and the room was dim and quiet during the experiment. Briefly, rats in all groups were placed in individual cylinders $(60 \mathrm{~cm}$ in height and $40 \mathrm{~cm}$ in diameter) containing water (depth $\left.40 \mathrm{~cm} ; 20 \pm 2{ }^{\circ} \mathrm{C}\right)$. The procedure included an initial 2-min swimming session without measurements and the latter 4-min duration of swimming. The total time of immobility in the latter 4-min duration of swimming was recorded by the software tracking and analysis system (Shanghai Xinsoft Information Technology Co., Ltd, Shanghai, China).

\section{Measurement of neurotransmitter levels}

After the behavioural tests, the rats were gradually anaesthetized with Zoletil $50(20 \mathrm{mg} / \mathrm{kg})$ via intraperitoneal injection (Virbac S. A, Carros, France); then, animals were sacrificed and decapitated $(33,34)$. The hippocampus was rapidly removed and carefully dissected on an ice plate. The tissues were immediately collected into labelled sterile tubes, frozen in liquid nitrogen and then stored at $-80{ }^{\circ} \mathrm{C}$ until the assays were performed. Then, the hippocampus samples were weighed and homogenized by sonication in a specified amount of normal saline (100 microliters/ 10 milligrams). The homogenate was kept at $4^{\circ} \mathrm{C}$ for $30 \mathrm{~min}$ and then centrifuged at $12,000 \mathrm{rpm}$ for $3 \mathrm{~min}$ at $4{ }^{\circ} \mathrm{C}$. The supernatant was collected, obtained and stored at $-20^{\circ} \mathrm{C}$ and then used to measure the neurotransmitters and $\mathrm{Ca}^{2+}$ levels in the hippocampus of all the groups by using assay kits.

The protein concentration of the collected supernatants was determined via the BCA protein assay kit (CWBIO, Beijing, China). The neurotransmitter concentrations of DA, NE, and 5-HT in the hippocampus were analysed using commercial ELISA kits (Beijing Liweiping Biological Technology Co., Ltd., Beijing, China). All experimental steps were performed according to the kit operation specifications. OD values were measured by a microplate reader.

\section{Assessment of MAO and $\mathrm{Ca}^{2+}$ levels}

The collected supernatant above was further used to measure the monoaminoxidase (MAO) and $\mathrm{Ca}^{2+}$ levels in the hippocampus of all groups $(33,34)$. The MAO and $\mathrm{Ca}^{2+}$ concentrations in the hippocampus were analysed using ELISA kits (Beijing Liweiping Biological Technology Co., Ltd., Beijing, China). 
Moreover, rat blood samples were collected and centrifuged at $3,000 \mathrm{rpm} \cdot \mathrm{min}^{-1}$ for $20 \mathrm{~min}$, and the blood sera were obtained and stored at $-80{ }^{\circ} \mathrm{C}$ for further measurement. Then, an ELISA kit was used to assess the serum $\mathrm{Ca}^{2+}$ concentrations according to the kit operation instructions (Beijing Liweiping Biological Technology Co., Ltd., Beijing, China). The OD values were measured by a microplate reader (Spectrauor, TECAN, Sunrise, Austria).

\section{Histopathological examination}

Haematoxylin-eosin staining (H\&E) and Nissl's staining were used to reveal the histopathological features and injures of the cerebral cortex and hippocampus neurons, mainly containing the hippocampus CA1, CA2, CA3, and CA4 regions, in CUMS-induced models (33-35),. Following the behavioural tests, the animals were anaesthetized with Zoletil 50 and immediately sacrificed. Then, the remaining four rats of each group were perfused through the heart with cold phosphate-buffered saline (PBS, $\left.4^{\circ} \mathrm{C}\right)$, followed by cold $4 \%$-paraformaldehyde $\left(4^{\circ} \mathrm{C}\right)$. Finally, the whole brain of each rat was carefully removed and placed in brain buffer containing $4 \%$ formaldehyde fixative for $\mathrm{H} \& \mathrm{E}$ staining, toluidine blue (Nissl's) staining, immunohistochemical staining analysis and immunofluorescence assays.

The samples from each whole brain were embedded in paraffin and dissected into 5 - $\mu \mathrm{m}$ slices sections; 5 - $\mu \mathrm{m}$ thick coronal sections were generated and mounted on slides. The sections were stained using $\mathrm{H} \& \mathrm{E}$ and toluidine blue according to the described standard protocol (36). The staining images were acquired using a light microscope (Leica, Wetzlar, Germany).

\section{Immunofluorescence of BDNF}

To explore the effects of GXDSF on BDNF expression in CUMS-induced rats, immunofluorescence staining was performed as previously described $(35,37,38)$. Briefly, after the micro slides were deparaffinised, dewatered, and restored with a citrate-EDTA antigen retrieval solution (P0086, Beyotime, Shanghai, China) for $20 \mathrm{~min}$ at $95^{\circ} \mathrm{C}$, they were cooled down and washed with PBS three time (10 min per time), and the nonspecific binding sites on the sections were blocked with $5 \%$ goat serum albumin at room temperature for $60 \mathrm{~min}$ and incubated overnight with the anti-BDNF antibody (ab108319, 1:500 in dilution) at $4{ }^{\circ} \mathrm{C}$. Subsequently, they were incubated with a TRITC-conjugated goat anti-rabbit $\operatorname{IgG}(\mathrm{Hb}+\mathrm{L})$ at a
1:100 dilution (CW0160, CWBIO, Beijing, China) for $2 \mathrm{~h}$ at room temperature, and then counterstained by DAPI $(5 \mu \mathrm{g} / \mathrm{mL})$ for $10 \mathrm{~min}$. Images were immediately observed using fluorescence microscopy (Leica, Germany Q9) with a $400 \mathrm{X}$ magnification. The fluorescence intensity was evaluated by ImageJ software (Media Cybernetics, USA).

\section{Immunobistochemistry of NGF}

Immunohistochemical staining was used to confirm the effects of GXDSF on NGF expression. The remaining procedure was performed as described previously with slight modification $(33,35,39)$. Briefly, after the micro slides were deparaffinised, dehydrated, and restored with a citrate-EDTA antigen retrieval solution (P0086, Beyotime, Shanghai, China) for $20 \mathrm{~min}$, they were washed, and the nonspecific binding sites on sections were blocked with $5 \%$ goat serum albumin at room temperature for $60 \mathrm{~min}$; the slides were then incubated overnight with the antiNGF antibody (ab6199, 1:500 in dilution) at $4{ }^{\circ} \mathrm{C}$. Finally, the slides were incubated in $\mathrm{DAB}$ solution for $15 \mathrm{~min}$ at $37^{\circ} \mathrm{C}$, counterstained by haematoxylin and mounted with a water-based mounting medium. The brain tissue images were acquired using a light microscope (Leica, Wetzlar, Germany) with 200x and 400x and were randomly selected for image analysis via ImageJ software (Media Cybernetics, USA).

\section{Western blot analysis}

Western blotting was performed as previously reported $(40,41)$. Based on the manufacturer's instructions, the hippocampal tissues were weighed and homogenized using a tissue protein extraction kit (CWBIO, Shanghai, China) supplemented with $1 \%$ proteases and a phosphatase inhibitor cocktail (CWBIO, Shanghai, China). After the homogenates were centrifuged at $12,000 \times \mathrm{g}$ for $15 \mathrm{~min}$ at $4{ }^{\circ} \mathrm{C}$, the supernatant samples (including total protein, nuclear and cytolymph proteins) were collected. Then, the protein concentration in the supernatant was determined by a BCA assay (CWBIO, Shanghai, China). Finally, the protein samples $(3.5 \mathrm{mg} / \mathrm{mL})$ were diluted with $5 \times$ SDS loading buffer (CWBIO, Shanghai, China), denatured in boiling water for $5 \mathrm{~min}$, and then stored at $-80{ }^{\circ} \mathrm{C}$ until use.

Protein samples were loaded onto the SDS-PAGE gel $(10-15 \%)$, separated electrophoretically, and transferred onto PVDF membranes (Millipore, Bedford, MA, USA). After blocking the nonspecific binding sites for $2 \mathrm{~h}$ in $5 \%$ 


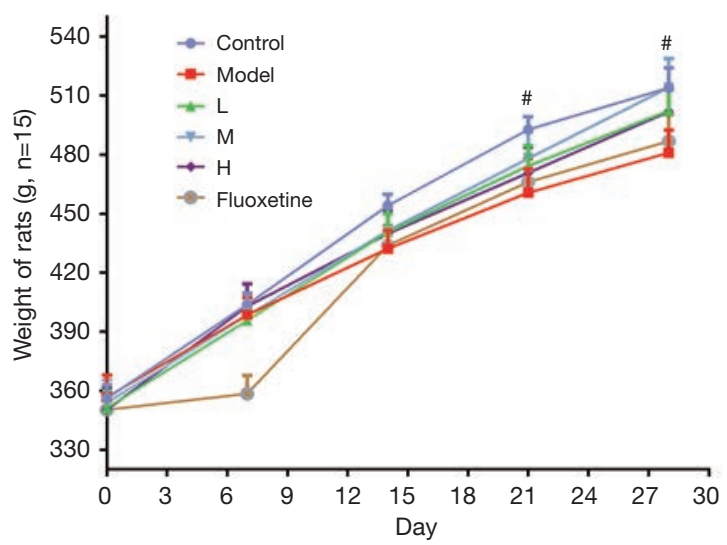

Figure 2 Effects of GXDSF on weight in the CUMS-induced rat model. GXDSF obviously inhibited the decrease in weight induced by CUMS. Data are presented as the mean values \pm standard error of the mean (SEM, $\mathrm{n}=15$ ); ${ }^{\text {P }}<0.05$ compared with the CUMS model group. CUMS means chronic unpredictable mild stress; GXDSF means Guanxin Danshen formula.

non-fat milk and Tris-buffered saline (TBS)/Tween 20 at room temperature, the membranes were individually incubated overnight at $4{ }^{\circ} \mathrm{C}$ with the following primary antibodies: BDNF (ab108319, 1:3,000, Abcam), NGF (ab6199, 1:2,000, Abcam), CaMKII (1:500, Santa Cruz, US), p-CaMKII (ab32678, 1:5,000, Abcam), p-CaMKII $\alpha$ (ab5683, 1:5,000, Abcam), p-CaMKII $\beta$ (ab124880, 5000, Abcam), CREB (ab32515, 1:3000, Abcam), p-CREB (ab32096, 1:3,000, Abcam), Syntaxin (ab188583, 1:5,000, Abcam) and $\beta$-actin $(1: 1,000)$. Then, the membrane was incubated at room temperature for $2 \mathrm{~h}$ with horseradish peroxidase conjugated antibodies at a 1:2,000 dilution. Protein expression was detected by an enhanced chemiluminescence method and imaged by using ChemiDoc XRS (Bio-Rad, Hercules, CA, USA). To eliminate variations in protein expression, three independent experiments were performed, and the data were adjusted to correspond to internal reference expression ( $\beta$-actin).

\section{Data and statistical analyses}

Data are presented as the mean values \pm standard error of the mean (SEM). All analyses were performed using GraphPad Prism 7.0 statistical software (GraphPad Software, Inc., La Jolla, San Diego, CA, USA). Two-way analysis of variance (ANOVA) was used with drug (GXDSF at $\mathrm{L}, \mathrm{M}$ and $\mathrm{H}$ doses $v s$. Vehicle) and treatment (CUMS $v s$. Control) as independent factors. Group differences after

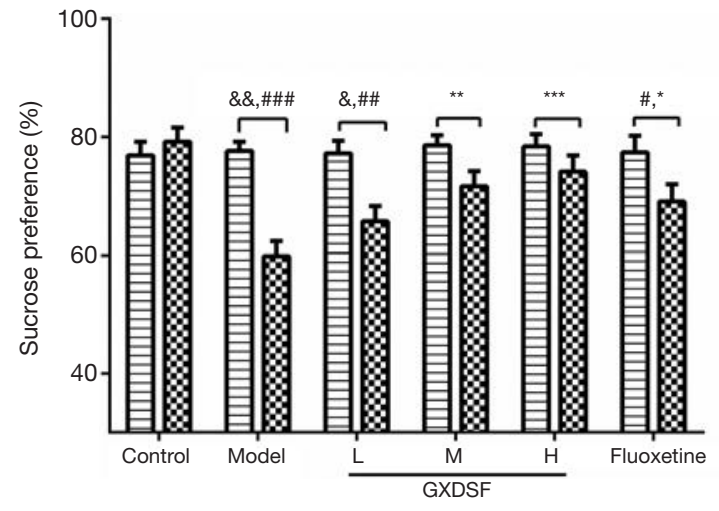

Figure 3 Effects of GXDSF on sucrose preference in the CUMSinduced rat model. GXDSF significantly increased sucrose preference in the CUMS-induced rat model. Data are presented as the mean values \pm standard error of the mean $(\mathrm{SEM}, \mathrm{n}=12)$; ${ }^{*} \mathrm{P}<0.05,{ }^{\& \&} \mathrm{P}<0.01$, day 0 vs. day $28 ;{ }^{*} \mathrm{P}<0.05,{ }^{* *} \mathrm{P}<0.01,{ }^{* * *} \mathrm{P}<0.001$ versus the model group on the $28^{\text {th }}$ day; ${ }^{\#} \mathrm{P}<0.05,{ }^{\# \#} \mathrm{P}<0.01$, ${ }^{\# \# \#} \mathrm{P}<0.001$ versus the control group on the 28 th day. CUMS, chronic unpredictable mild stress; GXDSF, Guanxin Danshen formula; SPT, sucrose preference test.

significant ANOVAs were measured by post hoc Bonferroni test, and $\mathrm{P}<0.05$ was considered statistically significant.

\section{Results}

\section{Effects of GXDSF on body weight}

First, during the 30-day CUMS induction, compared with the control group, the CUMS model rats had decreased body weight and showed significant inhibition from $492.8 \pm 20.7$ to $460.7 \pm 39.1 \mathrm{~g}$ on the 21 st day (Figure 2, $\mathrm{P}<0.05)$ and from $514.0 \pm 31.7$ to $481.0 \pm 36.3 \mathrm{~g}$ on the $28^{\text {th }}$ day (Figure 2, $\mathrm{P}<0.05$ ). Conversely, treatment with GXDSF (L, $\mathrm{M}$, and $\mathrm{H}$ ) improved body weight compared with the CUMS model but showed no significant difference from the control group. In addition, $2 \mathrm{mg} \cdot \mathrm{kg}^{-1}$ of fluoxetine treatment had a similar effect against depressant-like behaviours.

\section{Effects of GXDSF on the SPT}

As shown in Figure 3, the results of the sucrose preference test (SPT) were summarized to further investigate the antidepressant effects of GXDSF. Before the CUMS procedure, the baseline data from the sucrose preference presented no significant differences among the groups. After exposure to CUMS for 30 days, the sucrose preference was 

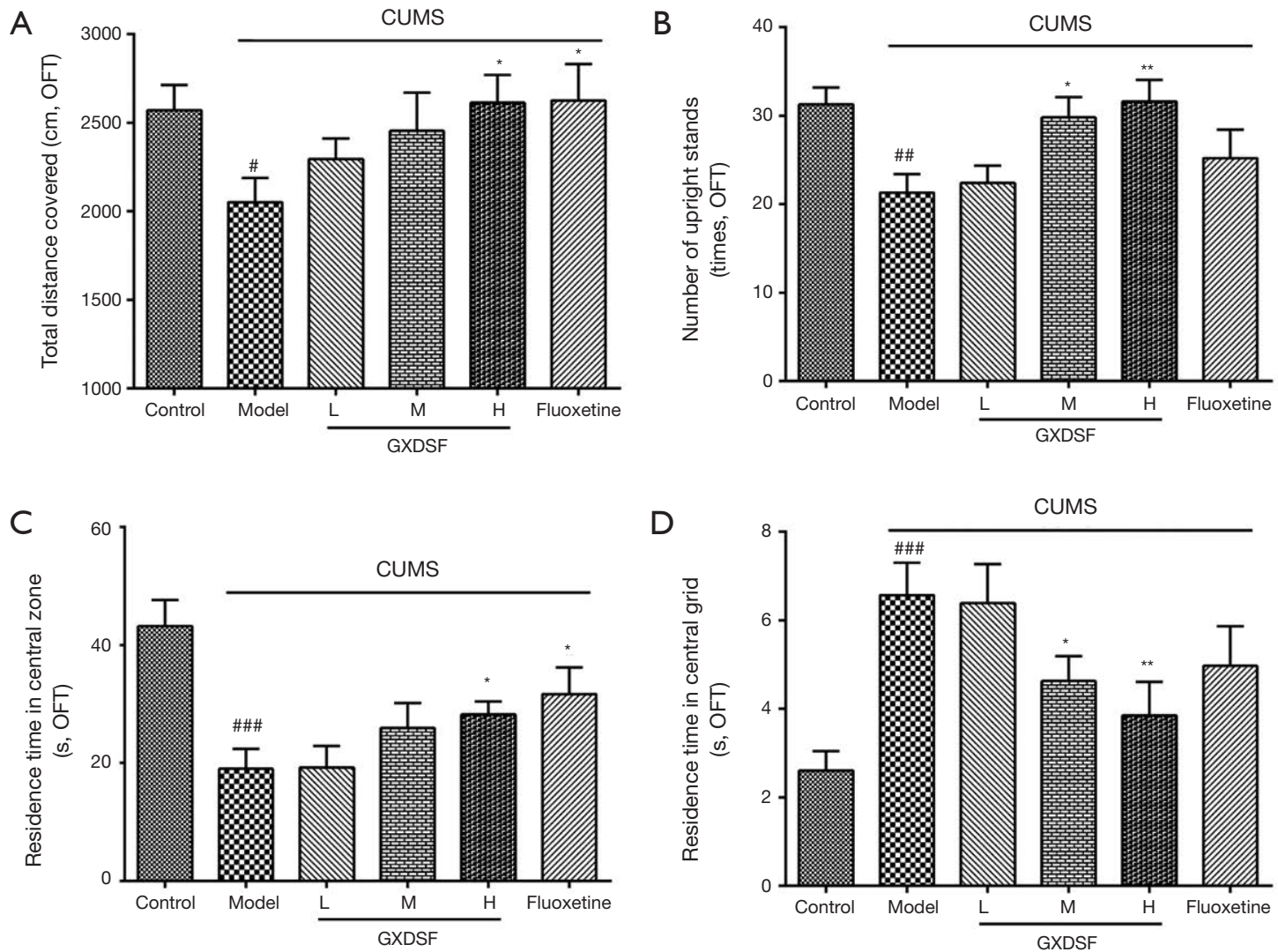

Figure 4 Effects of GXDSF on the open-field test in the CUMS-induced rat model. GXDSF ameliorated the depressant-like states induced by the CUMS model in the open-field test. (A) The total distance covered ( $\mathrm{cm}$ ) in 5 min in the open-field test; (B) the number of upright stands (times) in $5 \mathrm{~min}$ in the open-field test; (C) the central zone residence time (s) in 5 min in the open-field test; (D) the central grid residence time in $5 \mathrm{~min}$ in the open-field test (s). Data are presented as the mean values \pm standard error of the mean $(\mathrm{SEM}, \mathrm{n}=12)$; ${ }^{*} \mathrm{P}<0.05$, ${ }^{* *} \mathrm{P}<0.01$ compared with the model group; ${ }^{\#} \mathrm{P}<0.05,{ }^{\# \#} \mathrm{P}<0.01,{ }^{\# \#} \mathrm{P}<0.001$ compared with the control group. CUMS, chronic unpredictable mild stress; GXDSF, Guanxin Danshen formula; OFT, open-field test.

significantly reduced by CUMS induction, and the sucrose preference on the $28^{\text {th }}$ day showed a significant difference with that on the day 0 in the model group (Figure 3, $\mathrm{P}<0.01$ ) and the low-dose group (Figure 3, $\mathrm{P}<0.05$ ). Compared to the control group, the sucrose preference obviously decreased from $79.19 \% \pm 7.42 \%$ to $59.89 \% \pm 7.67 \%$ in the CUMS model group (Figure 3, $\mathrm{P}<0.001$ ); however, compared with the model group, treatment with low, moderate, and highdose GXDSF remarkably promoted the sucrose preference (Figure 3 Model, 59.89\% $\pm 7.67 \%$; L, 65.65\% $\pm 8.34 \%$; M, $71.61 \% \pm 8.09 \% ; \mathrm{H}, 74.07 \% \pm 8.82 \%)$ in a dose-dependent manner (Figure $3 \mathrm{P}<0.01$ and $\mathrm{P}<0.001$, respectively). In addition, $2 \mathrm{mg} \cdot \mathrm{kg}^{-1}$ of fluoxetine treatment had similar effects of greatly raising the sucrose preference (Figure 3, $\mathrm{P}<0.05)$. All of these studies demonstrated that GXDSF assessed an antidepressant-like effect.

\section{Effects of GXDSF on the OFT}

Following the SPT, the open-field test (OFT) was conducted, and the main results were analysed, including the total distance covered $(\mathrm{cm})$, the number of upright stands (times), the residence time in the central grid (s) and the central zone residence time (s). The total distance covered in OFTs is known to reflect the locomotor activity ability of animals. The residence time in the central zone and the number of upright stands reflect the depressivelike state of animals; a longer time spent in the central zones reflects a more depressive-like state. The central grid residence time in OFTs can reflect a decreased curiosity of the rat in the novel environment; a shorter time in the central grid can indicate greater curiosity $(3,42,43)$.

Figure 4 shows that in CUMS-induced rats, there 


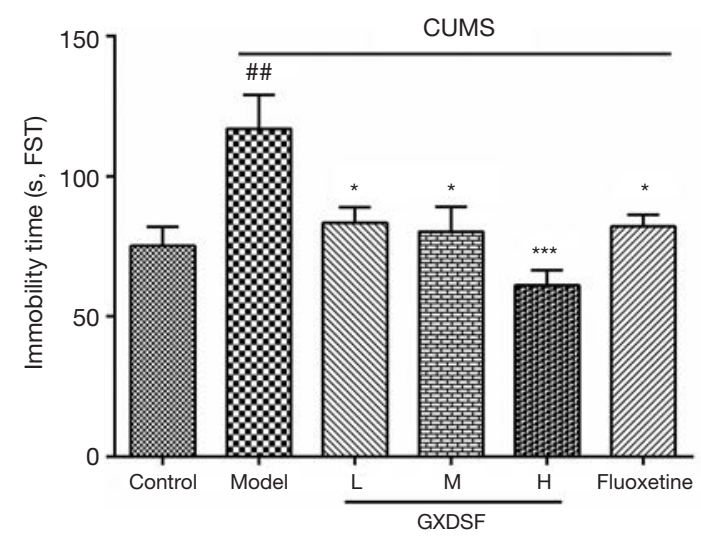

Figure 5 Effects of GXDSF on the immobility time in the TST in the CUMS-induced rat model. GXDSF decreased the immobility time in the open-field test. Data are presented as the mean values \pm standard error of the mean $(\mathrm{SEM}, \mathrm{n}=10) ;{ }^{*} \mathrm{P}<0.05,{ }^{* * *} \mathrm{P}<0.001$ versus the model group; ${ }^{\#} \mathrm{P}<0.01$ versus the control group. CUMS, chronic unpredictable mild stress; GXDSF, Guanxin Danshen formula; TST, tail suspension test.

were obvious differences in the total distance (from $25,708.6 \pm 4,519.4$ to $20,509.5 \pm 4,338.3 \mathrm{~mm}$ ) (Figure $4 A$, $\mathrm{P}<0.05$ ), the number of upright stands (from $31.3 \pm 5.9$ to $21.3 \pm 6.6$ ) (Figure $4 B, \mathrm{P}<0.01$ ), and the residence time in the central zone (from $43.2 \pm 14.0$ to $19.0 \pm 10.7$ s) (Figure $4 C$, $\mathrm{P}<0.001)$ compared to the control group. Luckily, the administration of GXDSF reversed the adverse effects caused by CUMS to some extent, meaning that, compared with the model group, different doses of GXDSF inhibited depressant-like behaviours in a dose-dependent manner in the OFT. The high-dose group showed a significant increase in the total distance (from 20,509.5 $\pm 4,338.3$ to $26,132.4 \pm 4,971.2 \mathrm{~mm}$ ) (Figure $4 A, \mathrm{P}<0.05$ ), the number of upright stands (from $21.3 \pm 6.6$ to $31.6 \pm 7.7$ ) (Figure $4 B$, $\mathrm{P}<0.01$ ), and the residence time in the central zone (from $19.0 \pm 10.7$ to $28.3 \pm 6.9$ s) (Figure $4 C, \mathrm{P}<0.05$ ) compared to the CUMS model group. Moreover, the central grid residence time in rats was adversely raised by CUMS model group, from $2.6 \pm 1.4$ to $6.6 \pm 2.3 \mathrm{~s}$ (Figure $4 D$, control $2.6 \pm 1.4 \mathrm{~s}$, model $6.6 \pm 2.3 \mathrm{~s}, \mathrm{P}<0.001$, respectively) but was remarkably reduced by GXDSF administration (Figure $4 D$, moderate, $4.6 \pm 1.8 \mathrm{~s}, \mathrm{P}<0.05$; high, $3.9 \pm 2.4 \mathrm{~s}$, $\mathrm{P}<0.01$, respectively). The low dose group increased in these indicators without significant differences. Analogously, $2 \mathrm{mg} \cdot \mathrm{kg}^{-1}$ of fluoxetine treatment also ameliorated the depressant-like states induced by the CUMS model in the open-field test.

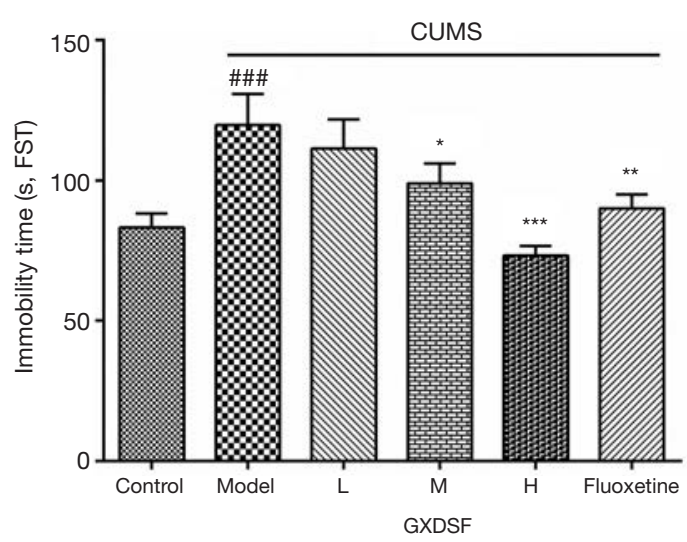

Figure 6 Effects of GXDSF on the immobility time in the FST in the CUMS-induced rat model. GXDSF decreased the immobility time in the open-field test. Data are presented as the mean \pm standard error of the mean (SEM, $\mathrm{n}=10)$ ) ${ }^{*} \mathrm{P}<0.05,{ }^{* *} \mathrm{P}<0.001$ versus the model group; ${ }^{\# \#} \mathrm{P}<0.001$ versus the control group. CUMS, chronic unpredictable mild stress; GXDSF, Guanxin Danshen formula; FST, tail suspension test forced swimming test.

\section{Effects of GXDSF on TST}

The immobility time in the TST was measured to further confirm the antidepressant-like effects of GXDSF. As shown in Figure 5, 30-day CUMS led to a marked increase in immobility time from $75.30 \pm 21.39$ to $117.0 \pm 38.0 \mathrm{~s}$ compared with the control group $(\mathrm{P}<0.01)$. By contrast, the low, moderate, and high doses of GXDSF treatment significantly decreased the immobility time of the CUMSinduced rats $(\mathrm{L}, 83.5 \pm 17.14 \mathrm{~s}, \mathrm{P}<0.05 ; \mathrm{M}, 80.3 \pm 27.67 \mathrm{~s}$, $\mathrm{P}<0.05 ; \mathrm{H}, 61.2 \pm 16.56 \mathrm{~s}, \mathrm{P}<0.001)$. Furthermore, $2 \mathrm{mg} \cdot \mathrm{kg}^{-1}$ of fluoxetine treatment also evidently reduced the immobility time in the CUMS-induced rat model (Figure 5, $\mathrm{P}<0.05)$

\section{Effects of GXDSF on the FST}

Similar to that of the tail suspension test above, GXDSF also showed remarkably antidepressant-like effects in the CUMS-induced rat model by increasing the immobility time in the FST.

Compared with the controls, CUMS-exposed rats manifested a significantly higher immobility, from $83.31 \pm 15.78$ to $119.7 \pm 35.04 \mathrm{~s}$, in the FST (Figure 6, $\mathrm{P}<0.001)$. In contrast, the low, moderate, and high doses of GXDSF treatment dose-dependently decreased the immobility time (Figure 6, L, 111.4 \pm 32.85 s, P>0.05; M, 

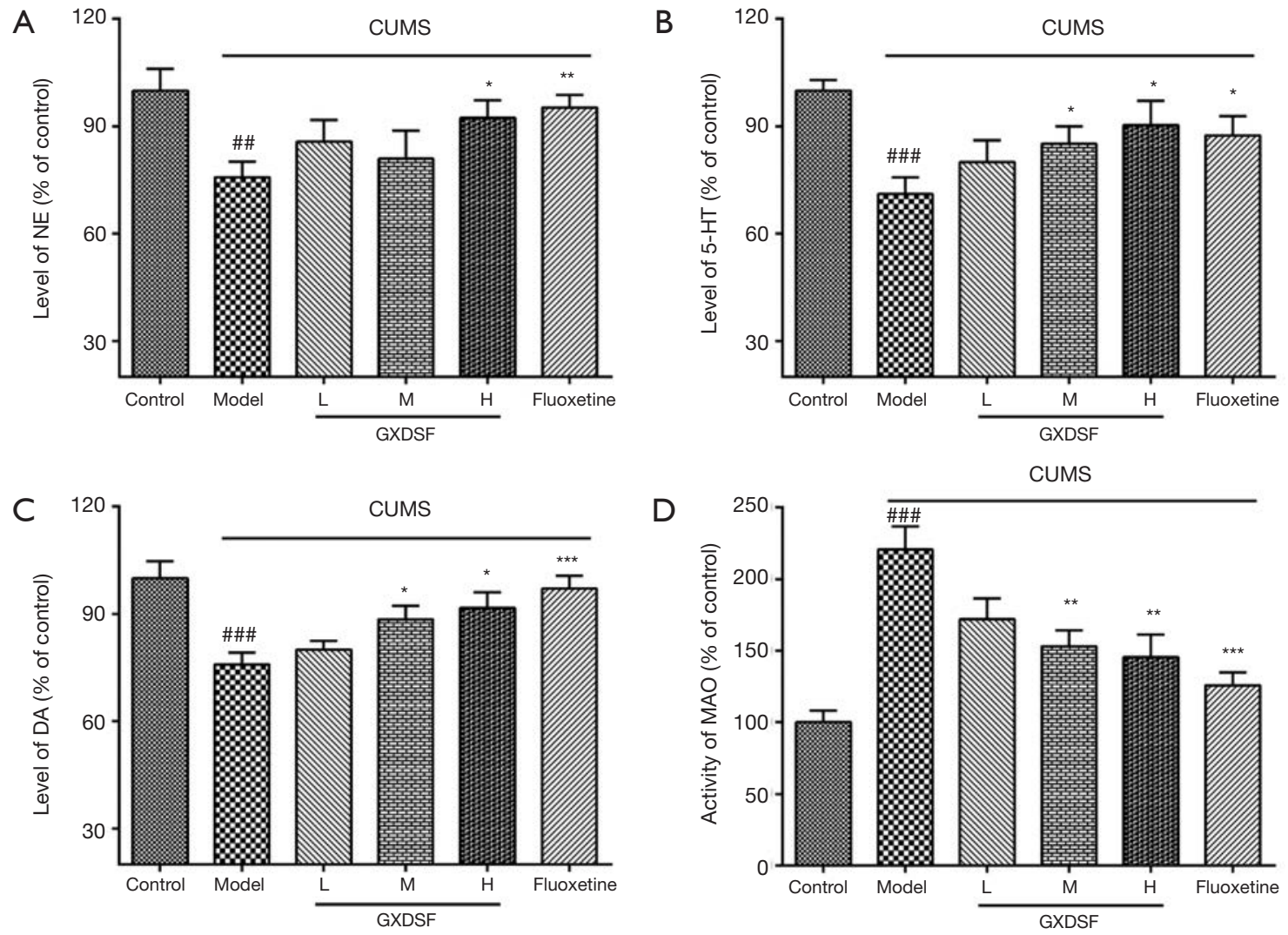

Figure 7 Effects of GXDSF on the monoamine neurotransmitters and monoaminoxidase activities in the CUMS-induced rat model. GXDSF upregulated the relative levels of neurotransmitters and inhibited the MAO activities in CUMS-induced rats. The levels of NE (A), 5-HT (B), DA (C) and the activities of MAO (D) in the hippocampus of CUMS-induced rats were detected by ELISA kits. Data are presented as the mean values \pm standard error of the mean (SEM, $\mathrm{n}=10$ ); ${ }^{*} \mathrm{P}<0.05,{ }^{* *} \mathrm{P}<0.01,{ }^{* * *} \mathrm{P}<0.001$ compared with the model group; ${ }^{\#} \mathrm{P}<0.05,{ }^{\# \#} \mathrm{P}<0.01,{ }^{\# \#} \mathrm{P}<0.001$ compared with the control group. CUMS, chronic unpredictable mild stress; GXDSF, Guanxin Danshen formula; MAO, monoaminoxidase.

$99 \pm 22.43$ s, $\mathrm{P}<0.05 ; \mathrm{H}, 73.3 \pm 10.46 \mathrm{~s}, \mathrm{P}<0.001)$ compared to the model group. In addition, $2 \mathrm{mg} \cdot \mathrm{kg}^{-1}$ of fluoxetine treatment also ameliorated the depressant-like states induced by the CUMS model in the FST.

\section{Effects of GXDSF on neurotransmitters and MAO}

It is known that depression is associated with the disturbance of brain neurotransmitters $(3,32)$. Hence, the neurotransmitter levels of DA, NE, and 5-HT in the hippocampus were analysed using commercial ELISA kits. After CUMS induction, the relative levels of $\mathrm{NE}$, 5-HT, and DA in the hippocampus of CUMS-induced rats were obviously decreased compared with the control group (Figure $7 A, \mathrm{P}<0.01$; Figure $7 B, \mathrm{P}<0.001$; Figure $7 C, \mathrm{P}<0.001)$. Luckily, the low, moderate, and high doses of GXDSF treatment dose-dependently inhibited the decrease in neurotransmitters to some extent, meaning that, compared with the model group, different doses of GXDSF ameliorated the depressant-like behaviours caused by CUMS. Compared with the CUMS model group, the relative levels of DA, NE, and 5-HT were significantly increased in the high dose group (Figure $7 A, \mathrm{P}<0.05$; Figure $7 B, \mathrm{P}<0.05$; Figure $7 C, \mathrm{P}<0.05)$. The moderate dose-treated groups displayed significant increases in the $5-\mathrm{HT}$ and DA concentrations (Figure $7 B, \mathrm{P}<0.05$; Figure $7 C, \mathrm{P}<0.05$, respectively). However, the differences were not significant in the low dose-treated groups.

Furthermore, the MAO activities in the hippocampus distinctly increased in the CUMS-induced rats (Figure $7 D, \mathrm{P}<0.001)$. Interestingly, the moderate and high doses of GXDSF may obviously restrain and downregulate the 

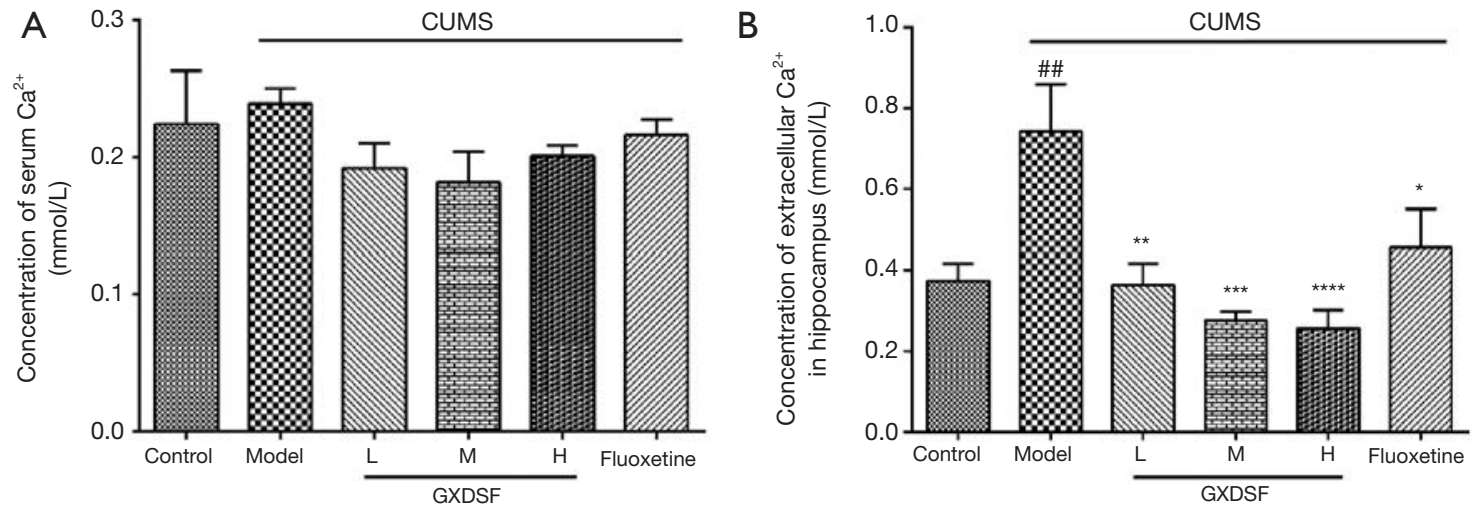

Figure 8 Effects of GXDSF on $\mathrm{Ca}^{2+}$ concentration in the CUMS-induced rat model. GXDSF upregulated the relative levels of Ca ${ }^{2+}$ concentration in CUMS-induced rats. (A) $\mathrm{Ca}^{2+}$ levels in the hippocampus; (B) $\mathrm{Ca}^{2+}$ levels in blood serum. Data are presented as the mean values \pm standard error of the mean $(\mathrm{SEM}, \mathrm{n}=10) ;{ }^{*} \mathrm{P}<0.05,{ }^{* *} \mathrm{P}<0.01,{ }^{* * *} \mathrm{P}<0.001,{ }^{* * *} \mathrm{P}<0.0001$ compared with the model group; ${ }^{* \#} \mathrm{P}<0.01$, compared with the control group. CUMS, chronic unpredictable mild stress; GXDSF, Guanxin Danshen formula.

MAO activities (Figure $7 D$, respectively, $\mathrm{P}<0.01 ; \mathrm{P}<0.01$ ), which could improve the neurotransmitter levels between synapses. Fluoxetine treatment evidently upregulated the relative levels of neurotransmitters and inhibited the MAO activities in CUMS-induced rats.

\section{Effects of GXDSF on $\mathrm{Ca}^{2+}$ concentration}

Evidence has also demonstrated the involvement of $\mathrm{Ca}^{2+}$ release in the pathophysiology of mood disorders $(44,45)$. Hence, the $\mathrm{Ca}^{2+}$ levels in the hippocampus and serum were analysed using commercial ELISA kits. After CUMS induction, the concentration of $\mathrm{Ca}^{2+}$ in the hippocampus of CUMS-induced rats increased notably, from $0.37 \pm 0.12$ to $0.74 \pm 0.33 \mathrm{mmol} / \mathrm{L}$, compared with the control group (Figure 8, $\mathrm{P}<0.001$ ). In contrast, different doses of GXDSF treatment dose-dependently decreased $\mathrm{Ca}^{2+}$ concentrations (Figure $8 B$ ). L, $0.36 \pm 0.15 \mathrm{mmol} / \mathrm{L}, \mathrm{P}<0.01$; $\mathrm{M}, 0.28 \pm 0.06 \mathrm{mmol} / \mathrm{L}, \mathrm{P}<0.001 ; \mathrm{H}, 0.26 \pm 0.13 \mathrm{mmol} / \mathrm{L}$, $\mathrm{P}<0.0001)$, meaning that GXDSF may ameliorate $\mathrm{Ca}^{2+}$ overload and $\mathrm{Ca}^{2+} /$ calmodulin complex production in hippocampal nerve cells, which may be one of the potential mechanisms of antidepressant-like behaviours. Moreover, fluoxetine treatment also evidently reduced the relative $\mathrm{Ca}^{2+}$ levels in CUMS-induced rats. The serum $\mathrm{Ca}^{2+}$ levels in each group showed no obvious difference (Figure $8 A$ ).

\section{Effects of GXDSF on histopathological features}

H\&E staining (Figures 9 and 10) showed that round and weakly stained nuclei of neurons were predominantly seen, and Nissl's staining showed that most neurons in exhibited weak staining; this finding indicated that neurons were diffusely deteriorated and that many Nissl bodies were lost in these neurons $(33,34)$. As shown in Figure $9 A$ and Figure $10 \mathrm{~A}$, the nerve cells of the control group presented a normal structural form: the cell outline was clear, the structure was compact, and the nucleolus was clearly visible. However, the morphological changes in the of neurons of the CUMSinduced rats were obvious in the cortex and the CA1, CA2, CA3 and CA4 regions of hippocampus compared to the control group (Figures 9 and 10); the gap around some neurons in the model groups was enlarged, the nerve cells were sparse, the neurons were swollen or shrunken, the nucleolus disappeared, the nuclear membrane was dissolved, and the nucleus was solid (marked with an arrow). Compared with the model group, the nerve cell changes were alleviated by GXDSF administration; only a few nerve cells were degenerated and irregularly scattered, and GXDSF reversed the observed phenomenon of diffusely deteriorated neurons and the loss of many Nissl bodies.

Compared with the control group, after CUMS induction, the nervous density (H\&E stained nuclei of neurons) in the hippocampus slides of the model groups showed significantly decreased in the CA1 region (from $262.63 \pm 52.83$ to $126.95 \pm 29.49, \mathrm{P}<0.0001$, Figure 9C), CA2 region (from $416.24 \pm 69.17$ to $174.02 \pm 35.39$, $\mathrm{P}<0.0001$, Figure 9D), CA3 region (from $300.41 \pm 72.45$ to $172.67 \pm 17.74, \mathrm{P}<0.001$, Figure 9E), and CA4 region (from $281.96 \pm 59.98$ to $175.75 \pm 56.92, \mathrm{P}<0.05$, Figure $9 F)$. However, 
A
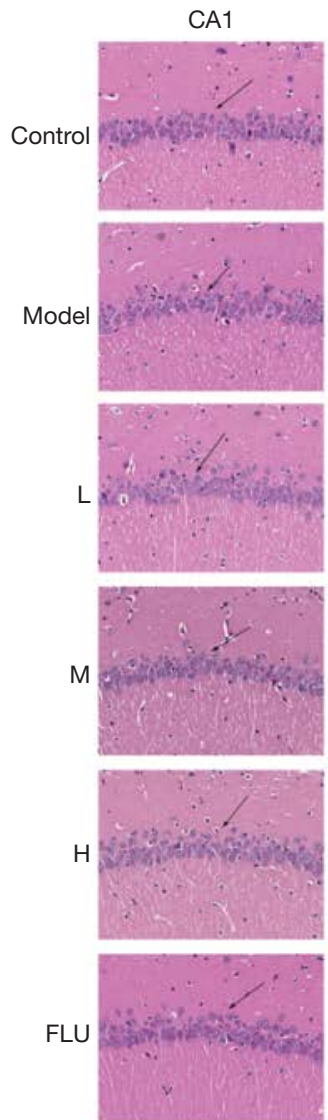

B

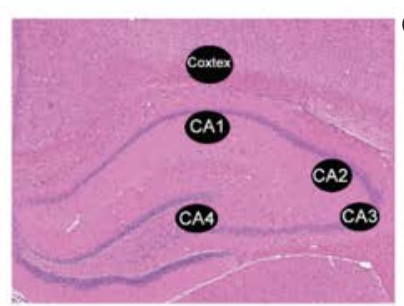

$\mathrm{E}$

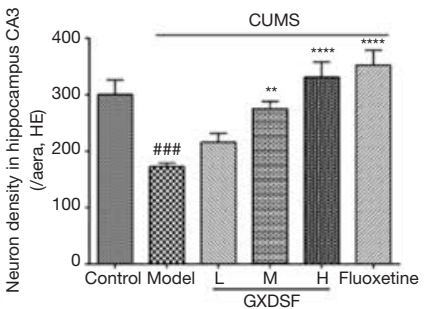

CA2
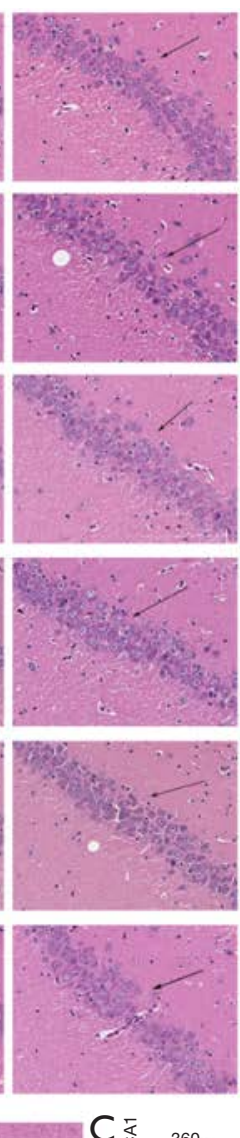

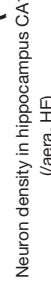

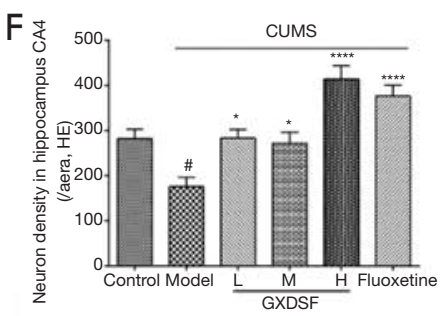

CA3
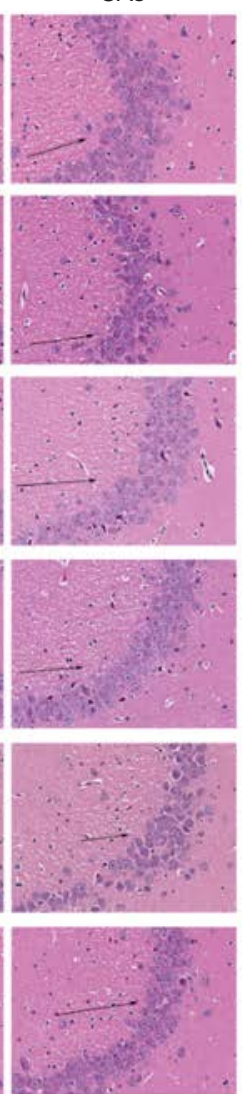
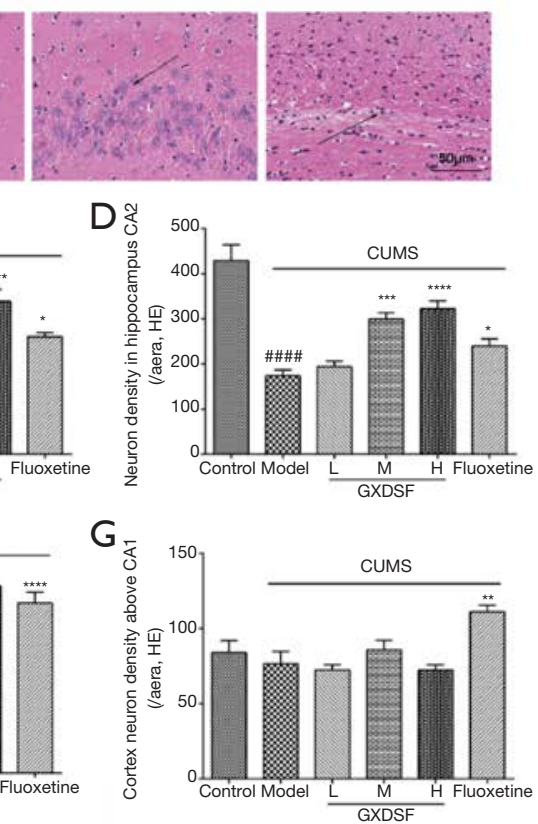

Figure 9 Representative images of H\&E staining performed on sections from depressant-like brain in the CUMS-induced rat model. (A) Representative H\&E staining images in the cortex and the CA1, CA2, CA3 and CA4 regions of the hippocampus in each group. (B) The image positions corresponding to the cortex, the CA1, CA2, CA3, and CA4 regions of the hippocampus in each group. (C) The nerve density in the CA1 region in each group. (D) The nerve density in the CA2 region in each group. (E) The nerve density in the CA3 region in each group. (F) The nerve density in the CA4 region in each group. (G) The nerve density in the cortex in each group. Data are presented as the mean values \pm standard error of the mean (SEM, $\mathrm{n}=6$ ); ${ }^{*} \mathrm{P}<0.05,{ }^{* *} \mathrm{P}<0.01,{ }^{* * *} \mathrm{P}<0.001,{ }^{* * * *} \mathrm{P}<0.0001$ compared with the model group; ${ }^{\#} \mathrm{P}<0.05,{ }^{\# \#} \mathrm{P}<0.001,{ }^{\# \#+"} \mathrm{P}<0.0001$ compared with the control group. Scale bar, $50 \mu \mathrm{m}$. CUMS, chronic unpredictable mild stress; GXDSF, Guanxin Danshen formula. The staining neurones are marked with black arrows. 
A
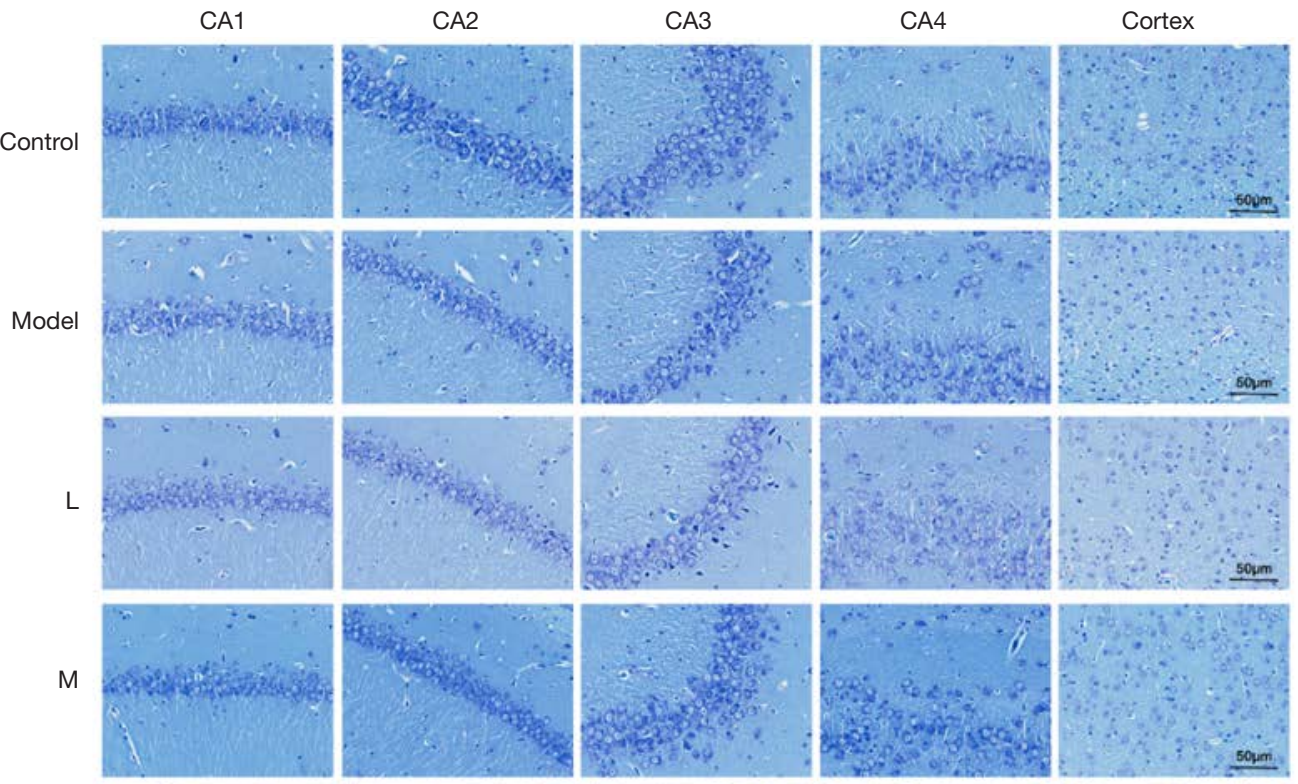

$\mathrm{H}$
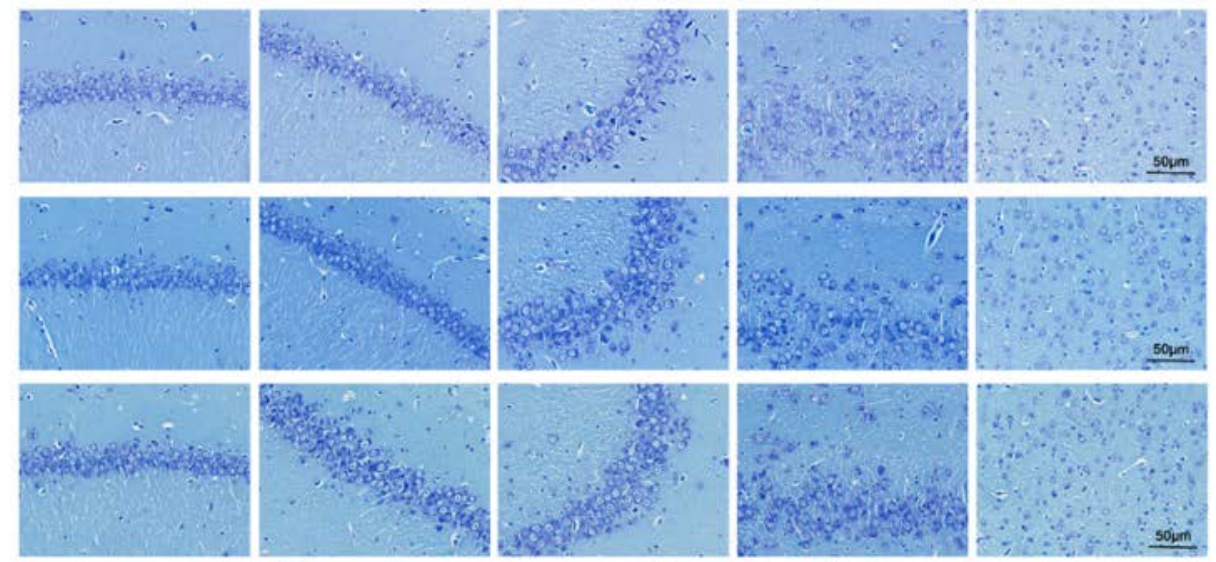

FLU
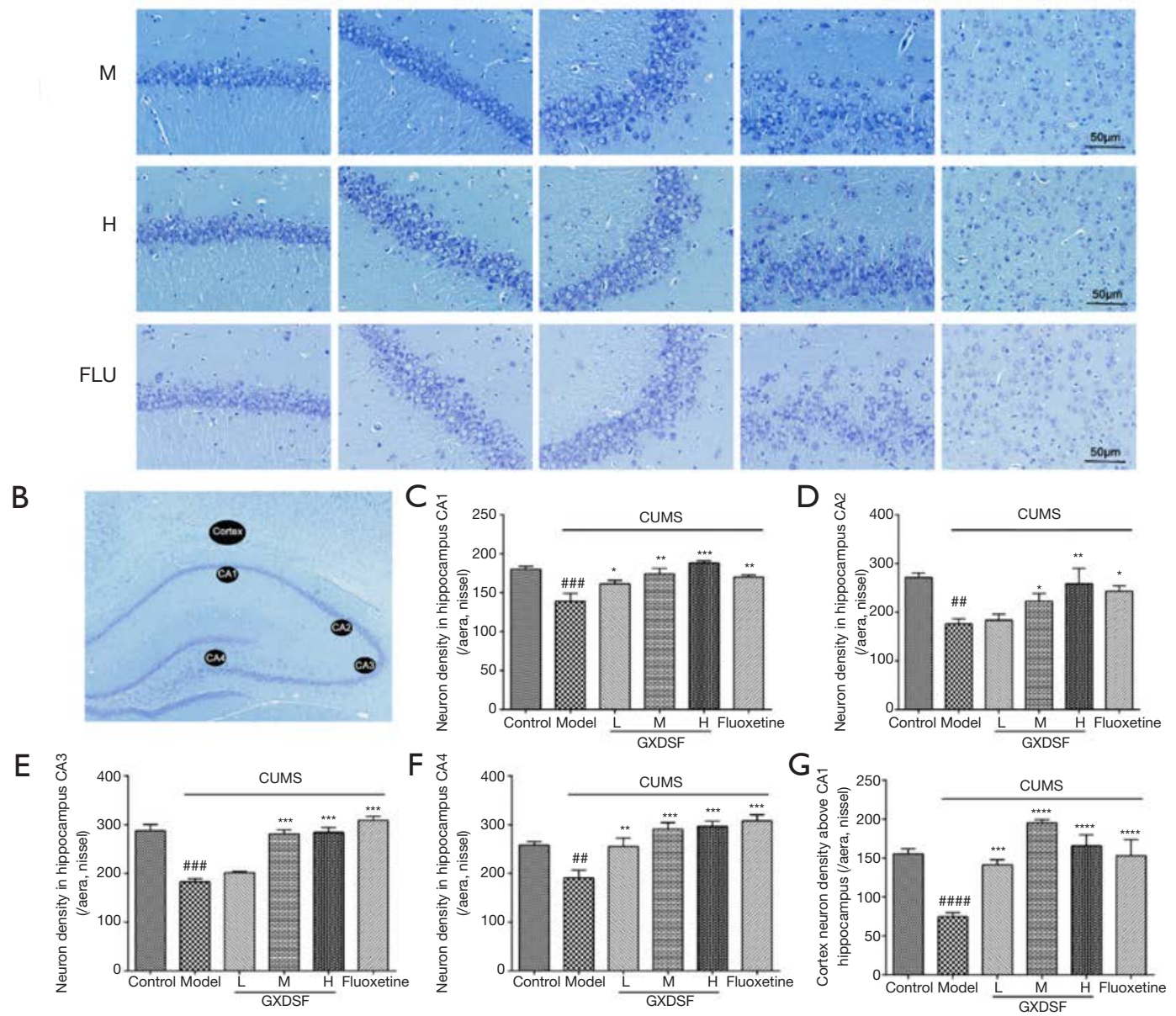

Figure 10 Representative images of Nissl's staining performed on sections from depressant-like brain in the CUMS-induced rat model. (A) Representative Nissl's staining images in the cortex and the CA1, CA2, CA3 and CA4 regions of the hippocampus in each group. (B) The image positions corresponding to the cortex, the CA1, CA2, CA3 and CA4 regions of the hippocampus in each group. (C) The nerve density in the CA1 region in each group. (D) The nerve density in the CA2 region in each group. (E) The nerve density in the CA3 region in each group. (F) The nerve density in the CA4 region in each group. (G) The nerve density in the cortex in each group. Images were analysed by Image J software. Data are presented as the mean values \pm standard error of the mean (SEM, $\mathrm{n}=6$ ); ${ }^{*} \mathrm{P}<0.05,{ }^{* *} \mathrm{P}<0.01,{ }^{* * *} \mathrm{P}<0.001$, ${ }_{* * * *} \mathrm{P}<0.0001$ compared with the model group; ${ }^{\# \#} \mathrm{P}<0.01,{ }^{\# \# \#} \mathrm{P}<0.001,{ }^{\# \# \#} \mathrm{P}<0.0001$ compared with the control group. Scale bar, $50 \mu \mathrm{m}$. CUMS, chronic unpredictable mild stress; GXDSF, Guanxin Danshen formula. 
the GXDSF treatment substantially and dose-dependently reduced the neuronal damage, the irregular scattering and the decrease in neuron density caused by CUMS, such that the neuron density of the high dose group demonstrated increased in the CA1 region (from $126.95 \pm 29.49$ to $244.14 \pm 54.18, \mathrm{P}<0.0001$, Figure $9 \mathrm{C}$ ), CA2 region (from $174.02 \pm 35.39$ to $281.357, \mathrm{P}<0.0001$, Figure 9D), CA3 region (from $172.67 \pm 17.74$ to $331.38 \pm 74.82, \mathrm{P}<0.0001$, Figure 9E), and CA4 region (from $175.75 \pm 56.92$ to $413.88 \pm 84.69, \mathrm{P}<0.0001$, Figure $9 F)$. In addition, the lowdose group exhibited a similar improvement in nervous density in the hippocampus regions, but these differences were not significant. The nervous density in the cortex showed no obvious change. Moreover, fluoxetine treatment also evidently inhibited the change in nervous density.

Nissl's staining revealed a change in Nissl bodies (neuron density). After CUMS induction, most neurons exhibited weak staining, which indicated that neurons were diffusely deteriorated, and many Nissl bodies and neurons were lost in the hippocampus neurons (Figure 10A and C, CA1, $\mathrm{P}<0.001$; Figure $10 D, \mathrm{CA} 2, \mathrm{P}<0.01$; Figure $10 \mathrm{E}$, CA3, $\mathrm{P}<0.001$; Figure 10F, CA4, $\mathrm{P}<0.01$; Figure $10 G$, cortex, $\mathrm{P}<0.0001)$. In contrast, the GXDSF-treated group exhibited strong staining and possessed neurons arranged regularly in the hippocampus. The high-dose group presented a significant increase in Nissl bodies and neuron density (Figure 10C, CA1, $\mathrm{P}<0.001$; Figure 10D, CA2, $\mathrm{P}<0.01$; Figure 10E, CA3, $\mathrm{P}<0.001$; Figure 10F, CA4, $\mathrm{P}<0.001$; Figure $10 G$, cortex, $\mathrm{P}<0.0001)$. In addition, the low- and moderate-dose groups exhibited similar improvements in nervous density in the hippocampus regions, with significant differences (Figure 10).

\section{Effects of GXDSF on BDNF and NGF}

The immunofluorescence assay showed that after a 30 day exposure to CUMS, the BDNF expression within the CA1 (Figure 11) and CA2 (Figure 12) regions was obviously decreased, but BDNF expression was improved by the low, moderate, and high doses of GXDSF administration in a dose-dependent manner (Figures 11 and 12). As shown in Figure $13 A$ and $B$, the relative fluorescence intensities of $\mathrm{BDNF}$ expression in the CA1 and CA2 regions in the model group were significantly decreased (Figure $13 A$, CA1, $\mathrm{P}<0.01$; Figure 13B, CA2, $\mathrm{P}<0.01)$. Conversely, GXDSF dosedependently and strikingly enhanced the relative fluorescence intensity of BDNF expression (Figure 13A, CA1, $\mathrm{P}<0.01$; Figure $13 B, \mathrm{CA} 2, \mathrm{P}<0.01)$ compared with the model group.
In addition, $2 \mathrm{mg} \cdot \mathrm{kg}^{-1}$ of fluoxetine treatment had similar effects, greatly increasing the relative fluorescence intensity of BDNF expression (Figure 13, $\mathrm{P}<0.01$ ). All of these studies demonstrated that GXDSF assessed neuroprotective and antidepressant-like effects via upregulating the expression of BDNF.

Similar to the BDNF expression assay, immunohistochemistry analysis revealed that the NGF relative levels within the cortex and CA2 regions (Figure 14) were distinctly reduced by a 30-day CUMS induction but were significantly increased by GXDSF administration (Figure 14). As shown in Figure 13C,D, the relative intensity of NGF expression in the cortex and CA2 regions in the model group was significantly decreased (Figure 13C, CA2, $\mathrm{P}<0.01$; Figure $13 D$, cortex, $\mathrm{P}<0.01)$. In contrast, the $\mathrm{L}$, $\mathrm{M}$, and $\mathrm{H}$ doses of GXDSF treatment strikingly increased the relative levels of NGF expression in a dose-dependent manner (Figure 13C, CA2, $\mathrm{P}<0.01$; Figure $13 \mathrm{D}$, cortex, $\mathrm{P}<0.01$ ) compared with the model group. Additionally, $2 \mathrm{mg} \cdot \mathrm{kg}^{-1}$ of fluoxetine treatment had a similar effect of greatly improving the relative levels of NGF expression (Figure 13, $\mathrm{P}<0.01$ ). All of these results suggested that GXDSF exerted neuroprotective and antidepressant-like effects via upregulating the expression of NGF.

\section{Effects of GXDSF on the expression levels of CaMKII and CREB/BDNF}

The results from previous studies have shown that the $\mathrm{Ca}^{2+}$ triggered activation of calcium/calmodulin-dependent protein kinases type II (CaMKIIs) are related to stressinduced depressive symptoms $(37,46-48)$. Based on the confirmed effects of GXDSF on hippocampus $\mathrm{Ca}^{2+}$ release in CUMS-induced rats, the related protein expression levels of CaMKII and its downstream CREB/BDNF signalling pathways in the hippocampus regions were investigated by Western blotting.

As shown in Figure 15A, the protein levels of CaMKII showed no obvious difference with a slight decline (Figure 15B), but the phosphorylated CaMKII (p-CaMKII) expression was significantly upregulated in the CUMS model group (Figure 15C, $\mathrm{P}<0.01$ ). Conversely, compared with model animals, treatment with GXDSF strikingly reduced the phosphorylation levels of total CaMKII protein in a dosedependent manner (Figure 15C, L, $\mathrm{P}<0.01 ; \mathrm{M}, \mathrm{P}<0.001$; $\mathrm{H}, \mathrm{P}<0.0001)$. Moreover, there were significant differences between the CUMS-induced rats and control rats with regard to the expression of the CaMKII $\alpha$ and CaMKII $\beta$ 

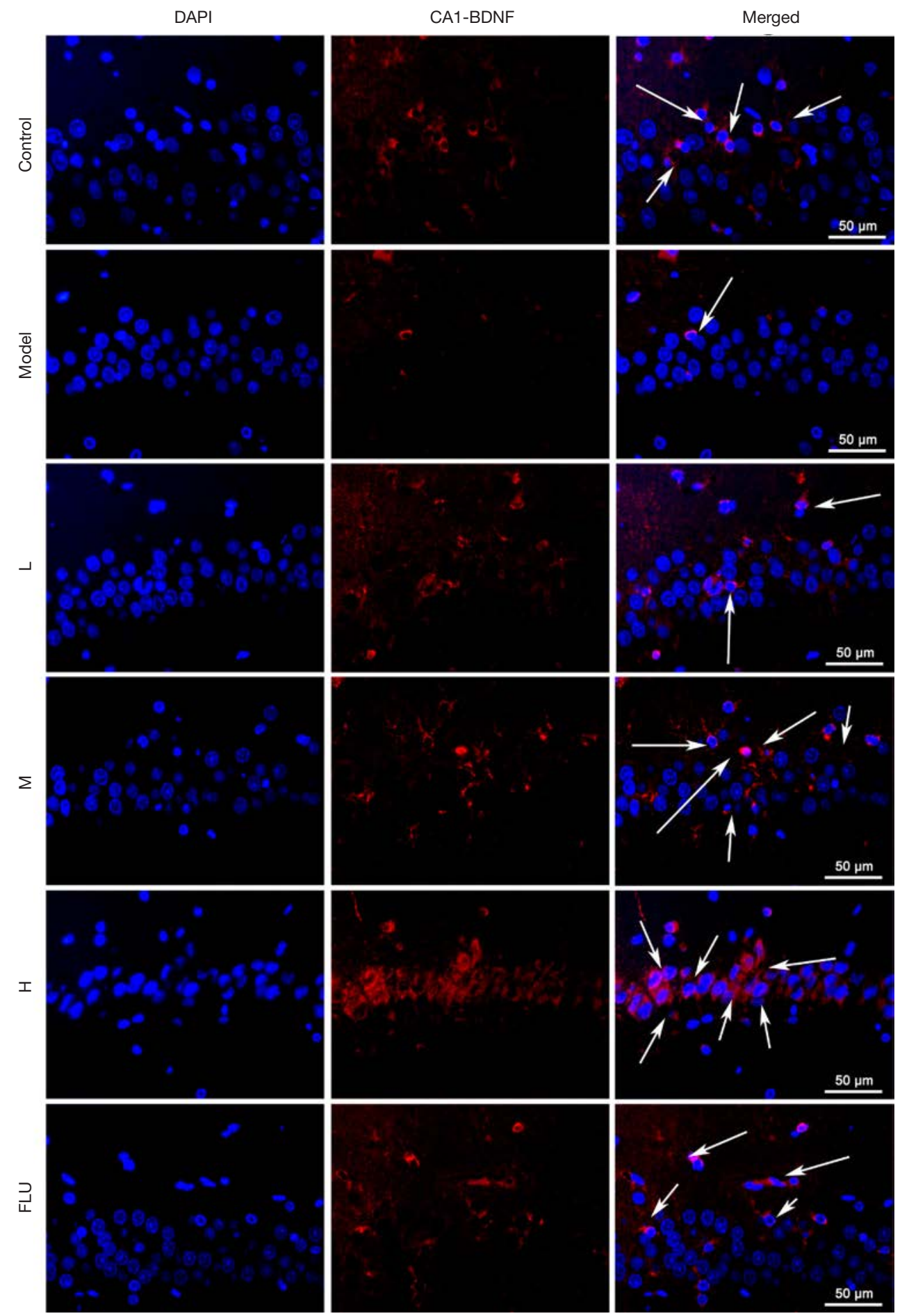

Figure 11 Effects of GXDSF on BDNF expression and representative images in the hippocampus CA1 region performed by immunofluorescence in the CUMS-induced rat model. Images revealed that GXDSF dose-dependently and strikingly enhanced the relative fluorescence intensity of CA1 region BDNF expression in CUMS-induced rats. Scale bar, $50 \mu \mathrm{m}$. The positive neurocytes are marked with white arrows, meaning that they were accompanied by high BDNF expression. 
DAPI
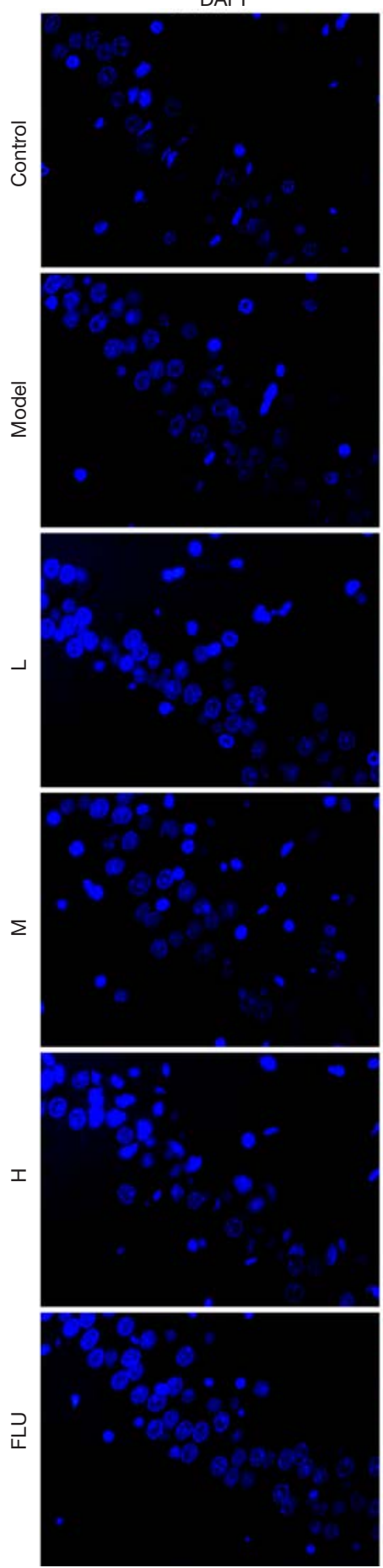

CA2-BDNF
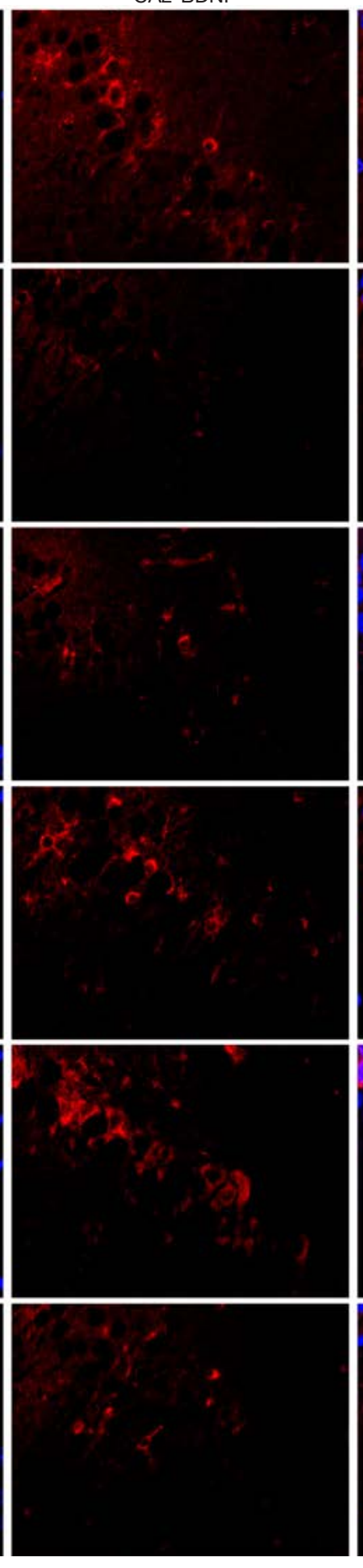

Merged
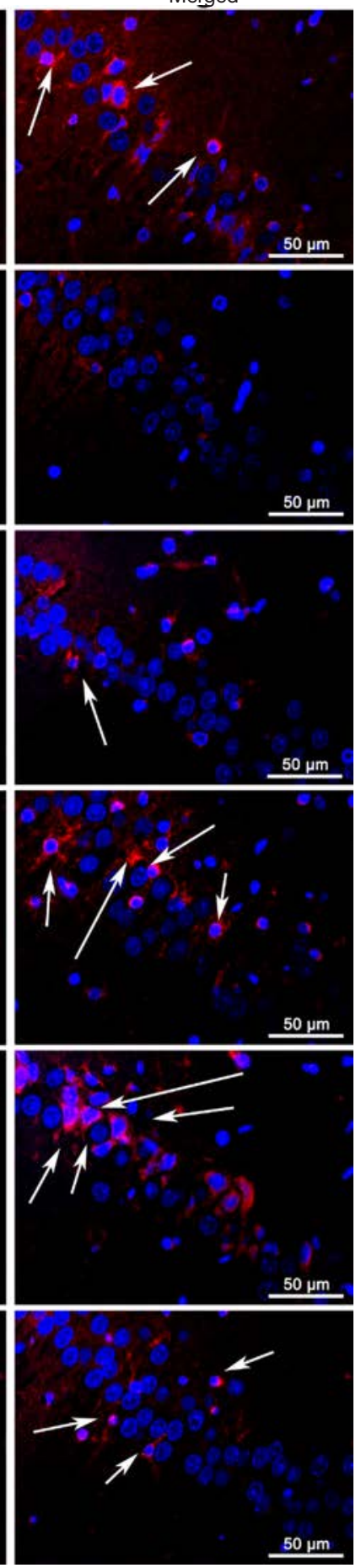

Figure 12 Effects of GXDSF on BDNF expression and representative images in the hippocampus CA2 region performed by immunofluorescence in the CUMS-induced rat model. Images showed that GXDSF dose-dependently and obviously improved the relative fluorescence intensity of CA2 region BDNF expression in CUMS-induced rats. Scale bar, $50 \mu \mathrm{m}$. The positive neurocytes are marked with white arrows, meaning that they are accompanied by high BDNF expression. 

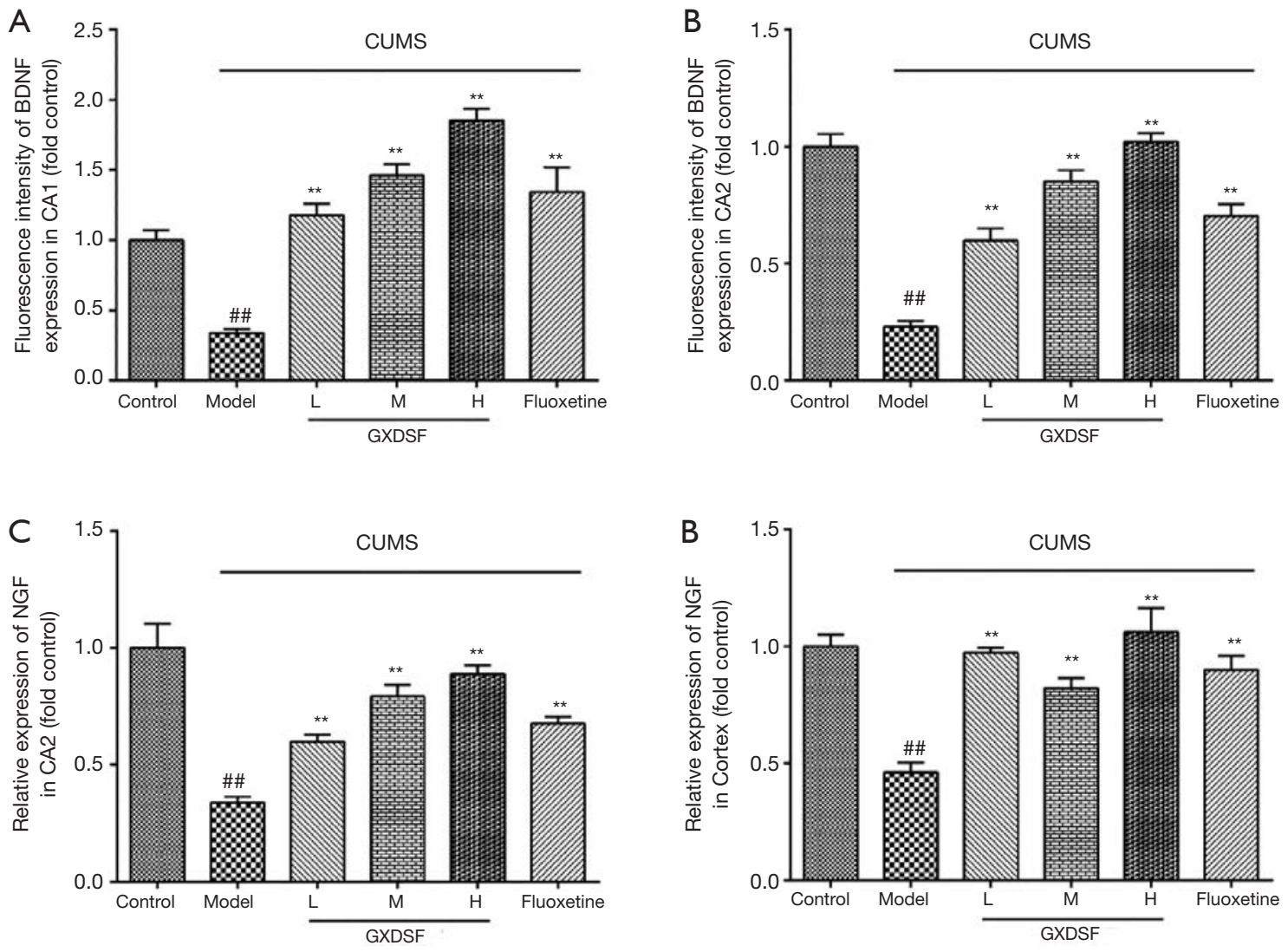

Figure 13 Relative fluorescence intensities of BDNF expression in the CA1 and CA2 regions and the relative levels of NGF expression in the cortex and CA2 regions in the CUMS-induced rat model. (A) The relative fluorescence intensities of BDNF expression in the hippocampus CA1 regions in each group. (B) The relative fluorescence intensities of BDNF expression in the CA2 region of the hippocampus in each group. (C) The relative intensity of NGF expression in the CA2 region of hippocampus. (D) The relative intensity of NGF expression in the cortex region of hippocampus. Images were analysed by ImageJ software. Data are presented as the mean values \pm standard error of the mean (SEM, $\mathrm{n}=4$ ); ${ }^{* *} \mathrm{P}<0.01$, compared with the model group; ${ }^{\#} \mathrm{P}<0.01$, compared with the control group. CUMS, chronic unpredictable mild stress; GXDSF, Guanxin Danshen formula.

proteins (Figure 15D). Compared to the control group, the CUMS group significantly improved the phosphorylation levels of CaMKII $\alpha$ (p-CaMKII $\alpha$, Figure 15E, P<0.01), but the GXDSF treatment clearly downregulated the increased expression level of p-CaMKII $\alpha$ in the hippocampus (Figure 15E, $\mathrm{L}, \mathrm{P}<0.01 ; \mathrm{M}, \mathrm{P}<0.01 ; \mathrm{H}, \mathrm{P}<0.01$ ). Similarly, the increased phosphorylation of the CaMKII $\beta$ protein induced by CUMS was strikingly abrogated by treatment with GXDSF in a dose-dependent manner (Figure 15F, L, $\mathrm{P}<0.05 ; \mathrm{M}, \mathrm{P}<0.01 ; \mathrm{H}, \mathrm{P}<0.001)$, indicating that GXDSF may produce an antidepressant-like effect by suppressing the phosphorylation levels of CaMKII and its subunits.

As shown in Figure 16A, the protein levels of CREB and its phosphorylated level (p-CREB) in rats with CUMS induction were significantly decreased in the model group (Figure $16 B, \mathrm{P}<0.01$; Figure $16 C, \mathrm{P}<0.01$ ). In contrast, the low, moderate, and high doses of GXDSF treatment dosedependently increased the expression levels of CREB (Figure $16 B, \mathrm{~L}, \mathrm{P}>0.05 ; \mathrm{M}, \mathrm{P}<0.0001 ; \mathrm{H}, \mathrm{P}<0.01)$ and significantly increased the phosphorylated level of CREB (Figure 16C, L, $<0.001 ; \mathrm{M}, \mathrm{P}<0.0001 ; \mathrm{H}, \mathrm{P}<0.0001)$, indicating that RSAE may regulate the expression levels of CREB/p-CREB to produce an antidepressant effect.

Similarly, the decline in BDNF and synitaxine-1 expression induced by CUMS was strikingly abrogated by treatment with GXDSF (Figure 16D,E, L, $\mathrm{P}<0.001$; $\mathrm{M}, \mathrm{P}<0.01 ; \mathrm{H}, \mathrm{P}<0.01)$. Moreover, GXDSF treatment could obviously improve synitaxine- 1 expression in a dose- 


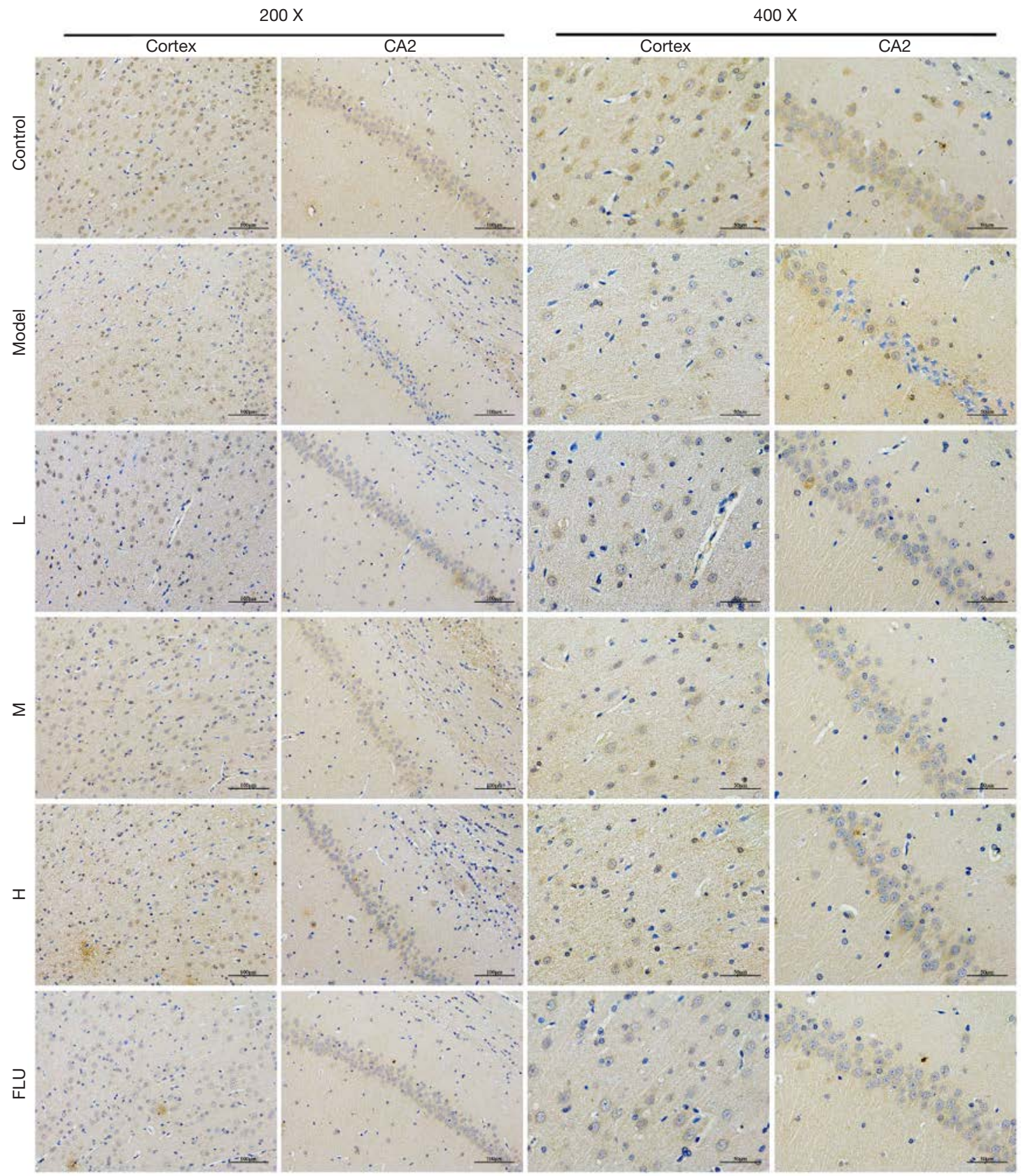

Figure 14 Effects of GXDSF on NGF expression and representative images in the cortex and hippocampus CA2 region performed by immunohistochemistry in the CUMS-induced rat model. Images suggest that GXDSF treatment distinctly increased the relative levels of NGF expression. Scale bar, $50 \mu \mathrm{m}$.

dependent manner (Figure 16D,F, L, $\mathrm{P}<0.01 ; \mathrm{M}, \mathrm{P}<0.01 ; \mathrm{H}$, $\mathrm{P}<0.01)$. These results indicated that RSAE may exert key protective and antidepressant-like effects against CUMSinduced depression in rats via regulating the CREB/BDNF signalling pathways.

\section{Discussion}

Depression is a chronic and recurrent syndrome of mood disorder causing an immense social and economic burden. 
A

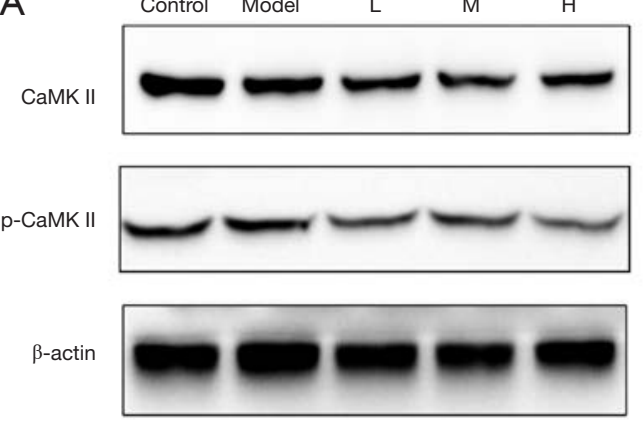

B

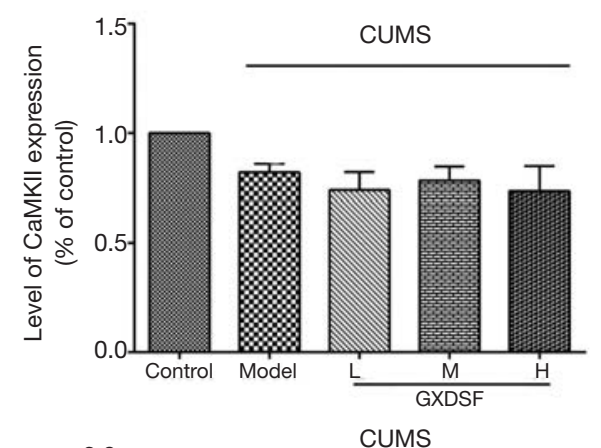

C

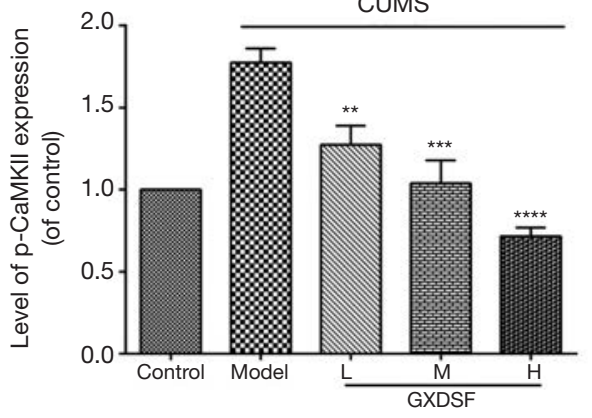

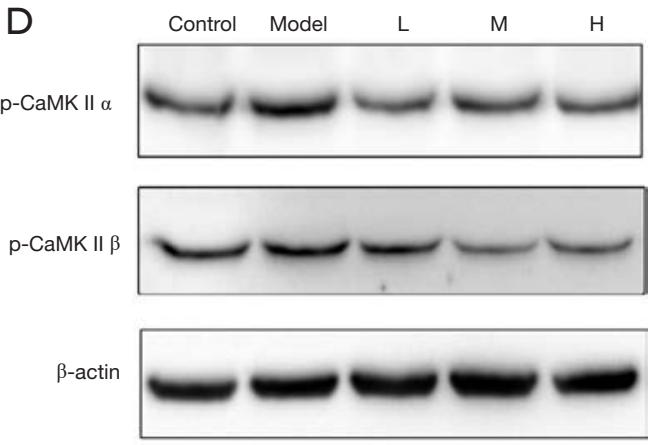

$\mathrm{E}$
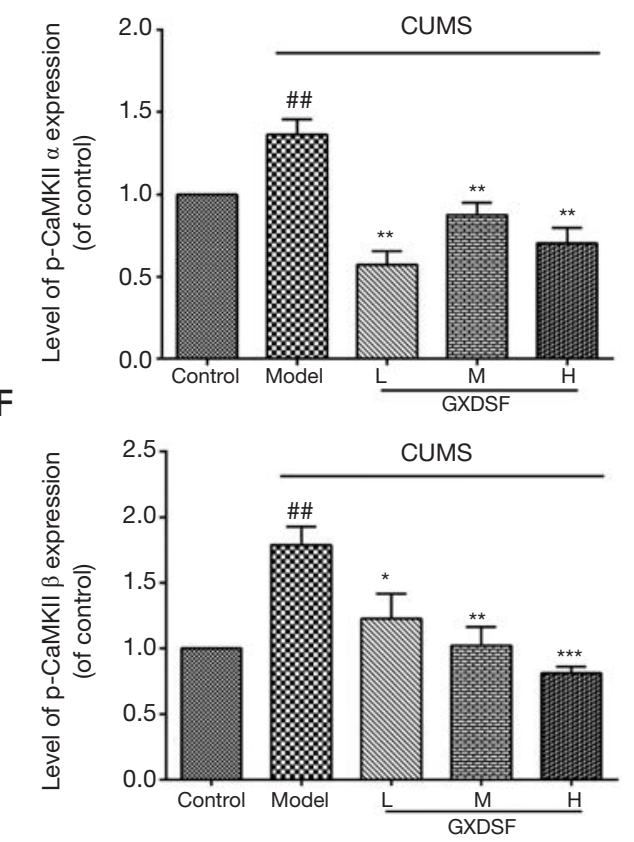

Figure 15 Effect of treatment with GXDSF on the p-CaMKII/CaMKII proteins in the CUMS-induced rat model. (A) The protein bands of p-CaMKII/CaMKII expression in the hippocampus. (B) The relative expression level of CaMKII in the hippocampus. (C) The relative expression level of p-CaMKII in the hippocampus. (D) The protein bands of p-CaMKII $\alpha$ and p-CaMKII $\beta$ expression in the hippocampus. (E) The relative expression level of p-CaMKII $\alpha$ in the hippocampus. (E) The relative expression level of p-CaMKII $\beta$ in the hippocampus. The IOD values of the proteins were quantified and analysed using Gel-Pro analyser software. Data are presented as the mean values \pm standard error of the mean (SEM, $\mathrm{n}=6$ ); ${ }^{*} \mathrm{P}<0.05,{ }^{* *} \mathrm{P}<0.01,{ }^{* * *} \mathrm{P}<0.001$, ${ }^{* * * *} \mathrm{P}<0.0001$ compared with the model group; ${ }^{{ }^{* \prime}} \mathrm{P}<0.01$ compared with the control group. CUMS, chronic unpredictable mild stress; GXDSF, Guanxin Danshen formula.

To date, the pathophysiology of depression is not clearly understood. Chronic stress is a major risk factor for depression. Chronic unpredictable mild stress (CUMS) is acknowledged as a model that mimics the state of stressinduced depression well and has been widely used in preclinical research with perfect aetiological, predictive and face validity $(49,50)$. Long-term unpredictable mild stresses lead to a series of depressant-like symptoms and behaviours, such as anxiety, despair, and cognitive impairment $(51,52)$.
Current animal studies have shown that the CUMS model is able to evoke depressive behavioural symptoms such as increased immobility and decreased sucrose consumption (50-53). In our study, after 30 days of the CUMS-induction procedure, the induced rats displayed a significant decrease in weight (Figure 2), sucrose consumption (Figure 3), the number of upright stands and the residence time of the central zone in the OFT (Figure 4), and a significant increase in immobility time in the FST (Figure 6) and TST 
A
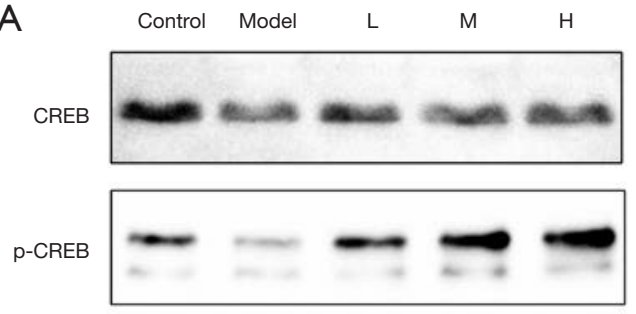

$\beta$-actin

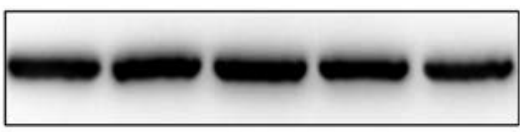

B

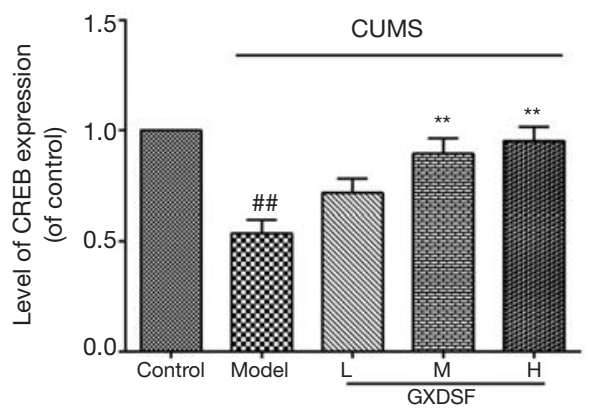

C

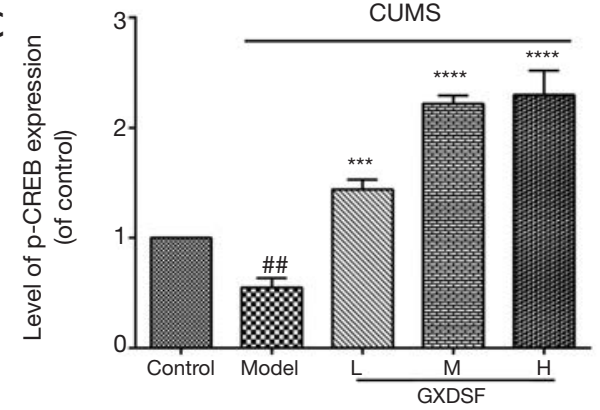

D
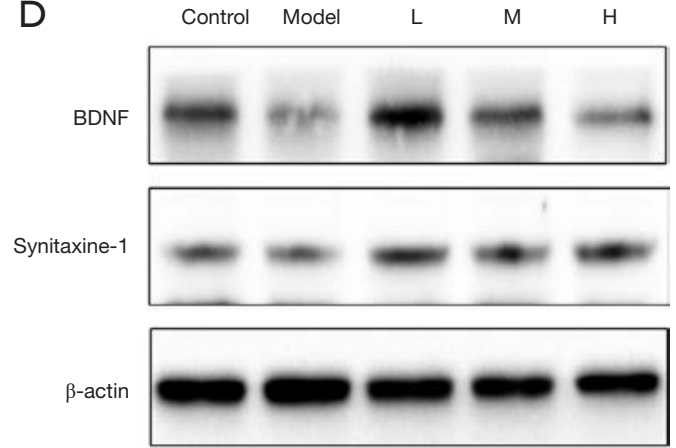

$\mathrm{E}$

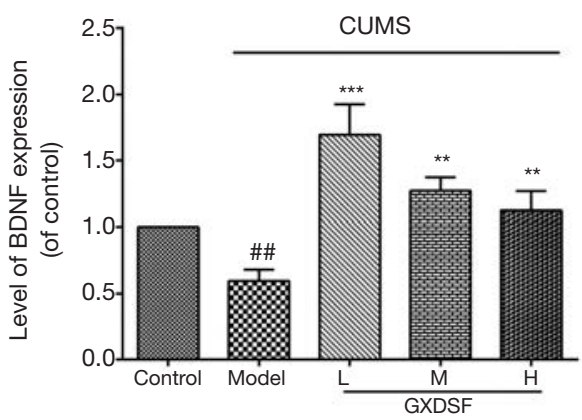

$\mathrm{F}$

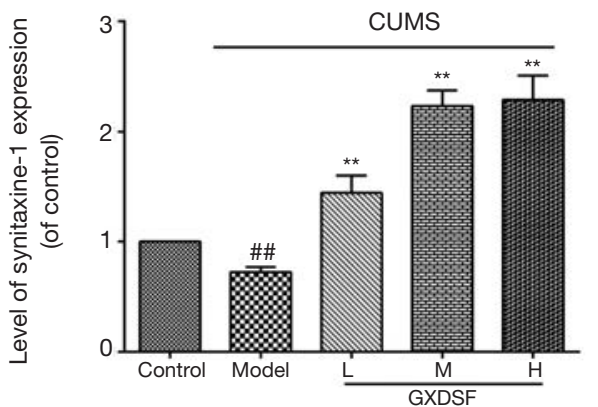

Figure 16 Effect of treatment with GXDSF on the CREB/BDNF signalling pathways in the CUMS-induced rat model. (A) The protein bands of p-CREB/CREB expression in the hippocampus. (B) The relative expression level of CREB in the hippocampus. (C) The relative expression level of p-CREB in the hippocampus. (D) The protein bands of BDNF and synitaxine-1 expression in the hippocampus. (E) The relative expression level of BDNF in the hippocampus. (F) The relative expression level of synitaxine-1 in the hippocampus. The IOD values of the proteins were quantified and analysed using Gel-Pro analyser software. Data are presented as the mean values \pm standard error of the mean $(\mathrm{SEM}, \mathrm{n}=6) ;{ }^{* *} \mathrm{P}<0.01,{ }^{* * *} \mathrm{P}<0.001,{ }^{* * * *} \mathrm{P}<0.0001$ compared with the model group; ${ }^{* \#} \mathrm{P}<0.01$ compared with the control group. CUMS, chronic unpredictable mild stress; GXDSF, Guanxin Danshen formula.

(Figure 5), in accordance with previous reports (3,19,32,4244,49,54), while GXDSF administration obviously reversed these alterations in a dose-dependent manner. All of these results proved that GXDSF may ameliorate these depression-like behaviours and exert antidepressant-like effects in CUMS-induced rats, similar to the effect observed with fluoxetine. Previously, GXDSF, a traditional Chinese formula, and its components, such as salvianolic acid $\mathrm{B}$ and ginsenoside $\mathrm{Rg} 1$, were reported to have cardio-protective effects against MIRI on cardiovascular disease (27), but these studies rarely involved investigations of neuroprotective effects against depressive disorders (27-31). Our study results found antidepressant-like effects of GXDSF, which could provide novel treatment strategies and clues for depression.

Depression is known to be closely associated with a low level of brain monoamines, including 5-HT, DA, and $\mathrm{NE}$, which has been widely recognized and evaluated 
$(21,55,56)$; monoamine oxidase (MAO-A/B) is an important enzyme in the metabolism of monoamines, presenting in neurons and astrocytes, that can inactivate monoamine neurotransmitters $(57,58)$. Therefore, the serotonergic, noradrenergic and dopaminergic systems play major roles in the effects of antidepressant-like drugs. Recent reports have also shown that, central monoamine neurotransmitters can be improved, particularly 5-HT, DA, and NE, after treatment with antidepressant drugs (49,54-56). In this study, CUMS induced monoamine deficiency through reduced levels of the monoamines 5-HT, DA and NE in the hippocampus (Figure 7, $\mathrm{P}<0.001$ ); in contrast, the levels of monoamine neurotransmitters were significantly increased by GXDSF treatment, as well as the positive control fluoxetine (Figure 7, $\mathrm{P}<0.05$ ). Moreover, GXDSF significantly inhibited the MAO activity increase, which reduced and suppressed the degradation and inactivation of monoamine neurotransmitters, in the hippocampus in CUMS-induced rats (Figure $7 D, \mathrm{P}<0.05$ ). These data were consistent with previous research (49,54-56) and suggested that the antidepressant effect of GXDSF might be partially due to the modulation of three major monoamine neurotransmitters, namely, 5-HT, DA and NE.

It is also widely acknowledged that BDNF and its mediated signalling pathways (its cognate receptor TrkB) participate in many behavioural and molecular mechanisms of antidepressants via alterations of synaptic plasticity, neuronal circuit formation, and neuronal survival (3,19-22). Recently, increasing evidence supports that BDNF expression is associated with the regulation of CREB and its phosphorylation levels (24,59-62), which has been reported to be a key mediator of cell survival and cognitive functions $(24,63)$. Our research results showed that CUMS exposure led to a decrease in BDNF in the hippocampus CA1 and $\mathrm{CA} 2$ regions (Figures $11,12, \mathrm{P}<0.01$ ) and NGF within the cortex and $\mathrm{CA} 2$ regions (Figure $13, \mathrm{P}<0.01$ ), consistent with previous reports (24,59-62). However, GXDSF dose-dependently and strikingly enhanced the relative expression levels of BDNF and NGF (Figure 14), which was similar to the effect observed with fluoxetine. Moreover, it was demonstrated that the CREB expression and its phosphorylation levels were reduced in the hippocampus of CUMS-induced rats (Figure 16, $\mathrm{P}<0.01$ ), while the chronic administration of GXDSF significantly reversed the decrease in CREB and p-CREB (Figure 16, $\mathrm{P}<0.01$ ). These results suggest that the increased levels of BDNF, NGF and CREB may explain the GXDSF-induced behavioural improvement effect in the CUMS model. Thus, this study provides the first in vivo demonstration of a possible mechanism of GXDSF via regulating changes in the CREBBDNF pathway.

Previous studies demonstrated that the direct phosphorylation of Ser133 in CREB was regulated by multiple upstream pathways and proteins $(62,64,65)$, such as the protein kinase A (PKA) (62,64), ERK1/2 (66,67), phosphoinositide 3-kinase/ protein kinase B (PI3K/AKT) (68), and CaMKII $(69,70)$ signalling pathways. In addition, evidence has accumulated for the involvement of calcium/calmodulin and intracellular $\mathrm{Ca}^{2+}$ in the pathophysiology of mood disorders $(45,47,48)$, indicating that a supraspinal increase in intracellular calcium contents was involved in the modulation of mood leading to a depressant-like effect in laboratory animals (45), and blocking $\mathrm{Ca}^{2+}$ release from intracellular stores provoked a decrease in the immobility time inducing an antidepressantlike effect $(45,47,48,71)$. Interestingly, GXDSF may dose-dependently decrease $\mathrm{Ca}^{2+}$ levels and inhibit the $\mathrm{Ca}^{2+}$ overload caused by CUMS in hippocampus. Taken together, based on the experimental results, we speculate that GXDSF may enhance the antidepressant-like effects by inhibiting $\mathrm{Ca}^{2+}$ levels and regulating the CaMKII signalling pathway.

It is well known that CaMKII plays a crucial role in the regulation of synaptic plasticity, glutamate receptors, and the phosphorylation of cyclic adenosine phosphate response element-binding protein (CREB), which are relevant to the major depressive disorder (45,71-73). Nonetheless, the pathophysiological processes in which CaMKII and the main subtypes, including CaMKII $\alpha$ and CaMKII $\beta$, may be involved in and play a major role are not still clearly known. In this study, we revealed that CUMS caused a widespread upregulation in the phosphorylation levels of total CaMKII, CaMKII $\alpha$ and CaMKII $\beta$ expression in the rat hippocampus (Figure $15, \mathrm{P}<0.01$ ), in agreement with previous results $(37,46,48,74)$; however, the increased phosphorylation of CaMKII, CaMKII $\alpha$ and CaMKII $\beta$ protein induced by CUMS was strikingly abrogated by treatment with GXDSF in a dose-dependent manner (Figure 15, $\mathrm{P}<0.01$ ), indicating that the neuroprotective effects of GXDSF upon the amelioration of the depression-like behaviours induced by CUMS may be closely associated with protein CaMKII-mediated phosphorylation. Furthermore, the autophosphorylation of the primary antibodies used in our results, including p-CaMKII, p-CaMKII $\alpha$, and p-CaMKII $\beta$, is on threonine 286 which allows the kinase to switch from a calmodulin-dependent to a calmodulin-independent state and is required for various cellular functions, including 

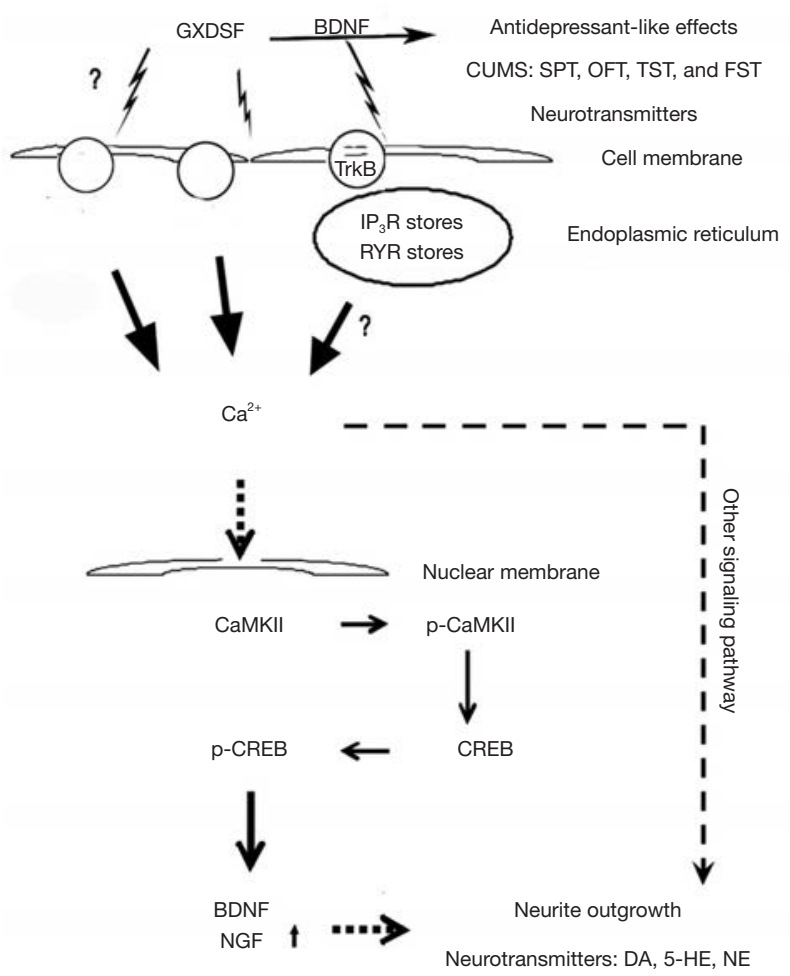

Figure 17 Antidepressant-like effects and mechanisms of GXDSF via mediation of the CaMKII-CREB-BDNF signalling pathway in chronic unpredictable mild stress (CUMS)-induced depressive rats. GXDSF, Guanxin Danshen formula.

hippocampal long-term potentiation, special learning, and hippocampus-dependent memory. In addition, CaMKII modified CREB binds to DNA efficiently as a monomer, which had been proved in the previous reports $(45,47,48,72-74)$. As summarized above, it was speculated that CREB was phosphorylated at serine 133 via regulation of CaMKII-mediated phosphorylation in the GXDSFtreated CUMS model rats, resulting in improved BDNF, NGF and synitaxine-1 expression, upregulated monoamine neurotransmitters, and ameliorated synaptic plasticity and neuronal circuit formation, which indicates that the CaMKII-CREB-BDNF signalling pathway plays a role in the antidepressant-like effect of GXDSF.

However, several limitations of this study still existed and should be noted. First, given the mixed preparation of natural medicinal plants composing GXDSF, the chemical components of each medical plant in the GXDSF preparation were explored by HPLC analysis, but the active pharmaceutical ingredients of the antidepressant effects have not completely been determined. Second, it is still unclear whether the pharmacokinetic and pharmacological interaction of multi-components in GXDSF might lead to its different effect traits and intensities in the antidepressantlike effects. Third, after GXDSF administration, changes in the regulation of the intracytoplasmic and intranuclear CREB/p-CREB levels were not clearly elaborated under the treatment of the CaMKII inhibitors, and the present study did not detect changes in the regulation of BDNF downstream, such as BDNF-TrkB and PI3K/AKT. Nevertheless, our study initially proved that GXDSF may be a potential candidate for development as an antidepressant for patients suffering from depressive disorders. Recent reports showed a significant sex difference regarding depression, and women reported more depressive symptoms than men $(75,76)$. Some meta-analytic summaries and reports also suggested that there was no sex difference, and within-sex variations were discussed as limitations to the emphasis on sex differences $(77,78)$. Although men and women share many psychological features, they also differ in important ways that can be understood in terms of evolutionary principles, primarily sexual selection and its extensions, and there are limitations of using animal studies to address human sex/gender neurobehavioural health disparities (78). Hence, the CUMS-induced male rat model was often used in animal experiments. If necessary, the CUMS-induced female rat model could be used to further explore the effects and mechanisms of GXDSF against depression.

\section{Conclusions}

In summary, our research initially proved that GXDSF exerts neuroprotective and antidepressant-like effects in CUMSinduced model rats (Figure 17). First, the summarized results demonstrate that GXDSF improved body weight, greatly raised the sucrose preference, significantly increased the total distance, number of upright stands, and central zone residence time in the OPF and reduced the immobility time in the TST and FST in CUMS model rats, indicating that GXDSF may ameliorate these depression-like responses and possess antidepressant-like effects. Second, GXDSF significantly upregulated the relative levels of neurotransmitters in a dose-dependent manner and inhibited the MAO activities in the hippocampal regions in CUMSinduced rats; additionally, GXDSF may dose-dependently decrease $\mathrm{Ca}^{2+}$ concentrations, inhibit $\mathrm{Ca}^{2+}$ overload and inhibit the upregulation of MAO activity caused by CUMS in the hippocampal nervous regions. All of these factors 
may be tightly associated with the potential mechanisms of antidepressant-like behaviours. Third, the regulated protein analyses revealed that GXDSF downregulated the phosphorylation levels of intracellular CaMKII, including both of its two subunits (CaMKII $\alpha$ and CaMKII $\beta$ ), and reversed the decline in intracellular CREB and p-CREB expression in the hippocampus, resulting in improved nuclear transcription activities of CREB, and thus, evident upregulation of the downstream effector protein expression levels of BDNF, NGF, and synitaxine-1 in the hippocampus of CUMS-induced rats, which contributed to synaptic plasticity, neuronal circuit formation, and neuronal survival against depression-related behaviour changes. In addition, Nissl staining and H\&E staining showed that GXDSF reversed the phenomenon that the neurons were diffusely deteriorated and that many Nissl bodies were lost in these neurons.

Hence, this study suggests that systemic administration of GXDSF may possess antidepressant-like effects in this CUMS-induced animal model. This study also provides the first in vivo demonstration for a possible mechanism of GXDSF via regulating changes in the CaMKII-CREBBDNF signalling pathway. These findings provide a novel potential substrate by which herbal antidepressants may exert therapeutic effects in the treatment of depression.

\section{Acknowledgments}

Funding: This research was supported by the Project "Study on Anti-anxiety and Anti-depression of Guanxin Danshen Pills", the Fundamental Research Funds for the Central Universities (Grant Number: 3332018152), the Beijing Natural Science Foundation (Grant Number: 7184229), the China Postdoctoral Science Foundation funded project (Grant Number: 2017M610063), the National Natural Science Foundation of China (Grant Number: No. 81503290), the Major Scientific and Technological Special Project for the Special Research Project for TCM (Grant Number: No. 201507004) and the National Natural Science Foundation of China (Grant Number: No. 81773938).

\section{Footnote}

Conflicts of Interest: The authors have no conflicts of interest to declare.

Ethical Statement: The authors are accountable for all aspects of the work and ensuring that questions related to the accuracy or integrity of any part of the work are appropriately investigated and resolved. The study was conducted in accordance with the Declaration of Helsinki. The protocol was approved by the Laboratory Animal Ethics Committee of the Institute of Medicinal Plant Development, Peking Union Medical College, and conformed to the Guide for the Care and Use of Laboratory Animals (Permit Number: SYXK 2017-0020).

\section{References}

1. Blazer DG, Kessler RC, Mcgonagle KA, et al. The prevalence and distribution of major depression in a national community sample: the National Comorbidity Survey. Am J Psychiatry 1994;151:979.

2. Murray CJ, Lopez AD. Alternative projections of mortality and disability by cause 1990-2020: Global Burden of Disease Study. Lancet 1997;349:1498-504.

3. Xu P, Wang KZ, Lu C, et al. Antidepressant-like effects and cognitive enhancement of the total phenols extract of Hemerocallis citrina Baroni in chronic unpredictable mild stress rats and its related mechanism. J Ethnopharmacol 2016;194:819-26.

4. Tao W, Yu D, Qiang S, et al. Liquiritigenin reverses depression-like behavior in unpredictable chronic mild stressinduced mice by regulating PI3K/Akt/mTOR mediated BDNF/TrkB pathway. Behav Brain Res 2016;308:177-86.

5. Benson C, Mifflin K, Kerr B, et al. Biogenic Amines and the Amino Acids GABA and Glutamate: Relationships with Pain and Depression. Mod Trends Pharmacopsychiatry 2015;30:67-79.

6. Jeon SW, Kim YK. Neuroinflammation and cytokine abnormality in major depression: Cause or consequence in that illness? World J Psychiatry 2016;6:283-93.

7. Oakes P, Loukas M, Oskouian RJ, et al. The neuroanatomy of depression: A review. Clin Anat 2017;30:44-9.

8. Li W, Ling S, Yang Y, et al. Systematic hypothesis for post-stroke depression caused inflammation and neurotransmission and resultant on possible treatments. Neuro Endocrinol Lett 2014;35:104-9.

9. Lin FB, Hou DR, Tang QP. Research progress of depression and the application of esketamine. Nan Fang Yi Ke Da Xue Xue Bao 2016;37:567-inside back cover.

10. Zhang H, Zhou Z, Chen Z, et al. Ginsenoside Rg3 exerts anti-depressive effect on an NMDA-treated cell model and a chronic mild stress animal model. J Pharmacol Sci 2017;134:45-54.

11. Allison DJ, Ditor DS. The common inflammatory etiology 
of depression and cognitive impairment: a therapeutic target. J Neuroinflammation 2014;11:151.

12. Anderson G, Maes M. Neurodegeneration in Parkinson's disease: interactions of oxidative stress, tryptophan catabolites and depression with mitochondria and sirtuins. Mol Neurobiol 2014;49:771-83.

13. Anderson G, Maes M. TRYCAT pathways link peripheral inflammation, nicotine, somatization and depression in the etiology and course of Parkinson's disease. CNS Neurol Disord Drug Targets 2014;13:137-49.

14. Anderson G, Seo M, Berk M, et al. Gut Permeability and Microbiota in Parkinson's Disease: Role of Depression, Tryptophan Catabolites, Oxidative and Nitrosative Stress and Melatonergic Pathways. Curr Pharm Des 2016;22:6142-51.

15. Braga J, Campar A. Biological causes of depression in Systemic Lupus Erythematosus. Acta Reumatol Port 2014;39:218-26.

16. Cui J, Jiang L, Xiang H. Ginsenoside Rb3 exerts antidepressant-like effects in several animal models. J Psychopharmacol 2012;26:697-713.

17. Albert PR, Fiori LM. Transcriptional dys-regulation in anxiety and major depression: 5-HT1A gene promoter architecture as a therapeutic opportunity. Curr Pharm Des 2014;20:3738-50.

18. Liu MY, Zhang LJ, Liu MC. Effect of ginsenoside pretreatment on 5-hydroxytryptamine system in SD rats with myocardial infarction and depression. Zhonghua Nei Ke Za Zhi 2016;55:700-4.

19. Zhang Y, Gu F, Chen J, et al. Chronic antidepressant administration alleviates frontal and hippocampal BDNF deficits in CUMS rat. Brain Res 2010;1366:141-8.

20. Pechlivanova D, Tchekalarova J, Nikolov R, et al. Dose-dependent effects of caffeine on behavior and thermoregulation in a chronic unpredictable stress model of depression in rats. Behav Brain Res 2010;209:205-11.

21. aan het Rot M, Mathew SJ, Charney DS. Neurobiological mechanisms in major depressive disorder. CMAJ 2009; 180:305-13.

22. Haghighi M, Salehi I, Erfani P, et al. Additional ECT increases BDNF-levels in patients suffering from major depressive disorders compared to patients treated with citalopram only. J Psychiatr Res 2013;47:908-15.

23. Lee B, Shim I, Lee H, et al. Effect of ginsenoside Re on depression- and anxiety-like behaviors and cognition memory deficit induced by repeated immobilization in rats. J Microbiol Biotechnol 2012;22:708-20.

24. Liu Z, Qi Y, Cheng Z, et al. The effects of ginsenoside
$\mathrm{Rg} 1$ on chronic stress induced depression-like behaviors, BDNF expression and the phosphorylation of PKA and CREB in rats. Neuroscience 2016;322:358-69.

25. Si Y, Zhu J, Huang X, et al. Effects of Panax notoginseng saponins on proliferation and differentiation of rat embryonic cortical neural stem cells. J Chin Med Assoc 2016;79:256-63.

26. Zhao M, Chen L, Yang J, et al. BDNF Val66Met polymorphism, life stress and depression: A metaanalysis of gene-environment interaction. J Affect Disord 2018;227:226-35.

27. Deng X, Xing X, Sun G, et al. Guanxin Danshen Formulation Protects against Myocardial Ischemia Reperfusion Injury-Induced Left Ventricular Remodeling by Upregulating Estrogen Receptor $\beta$. Front Pharmacol 2017;8:777.

28. Sun G, Huang C, Lu Y, et al. Study on activating blood and eliminating stasis of guanxin dansheng capsule. Zhong Yao Cai 2002;25:119-21.

29. Zeng X, Yang J, Yang X, et al. Effect of Guanxin No.2 decoction on gene expression in different areas of the myocardial infarcted heart of rats using microarray technology. J Pharm Pharmacol 2009;61:213-9.

30. Yao H, Huang X, Li S, et al. Simultaneous Determination of Eight Phenolic Acids, Five Saponins and Four Tanshinones for Quality Control of Compound Preparations Containing Danshen-Sanqi Herb-pair by HPLC-DAD. Pharmacogn Mag 2017;13:64-75.

31. Tang SM, Xing XY, Deng XH, et al. Research progress of Guanxin Danshen formula and its effective components in treating coronary artery heart disease. Zhongguo Zhong Yao Za Zhi 2016;41:3721-6.

32. Jiang N, Zhang BY, Dong LM, et al. Antidepressant effects of dammarane sapogenins in chronic unpredictable mild stress-induced depressive mice. Phytother Res 2018;32:1023-9.

33. Meng X, Xie W, Xu Q, et al. Neuroprotective Effects of Radix Scrophulariae on Cerebral Ischemia and Reperfusion Injury via MAPK Pathways. Molecules 2018;23. doi: 10.3390/molecules23092401. Erratum in: Molecules 2018;23. doi: 10.3390/molecules23102704.

34. Zhai Y, Meng X, Luo Y, et al. Notoginsenoside R1 ameliorates diabetic encephalopathy by activating the Nrf2 pathway and inhibiting NLRP3 inflammasome activation. Oncotarget 2018;9:9344-63.

35. Tao X, Sun X, Yin L, et al. Dioscin ameliorates cerebral ischemia/reperfusion injury through the downregulation of TLR4 signaling via HMGB-1 inhibition ?. Free Radic 
Biol Med 2015;84:103-15.

36. Li Y, Yan J, Zhu X, et al. Increased hippocampal fissure width is a sensitive indicator of rat hippocampal atrophy. Brain Res Bull 2018;137:91-7.

37. Ahmed ME, Dong Y, Lu Y, et al. Beneficial Effects of a CaMKII $\alpha$ Inhibitor TatCN21 Peptide in Global Cerebral Ischemia. J Mol Neurosci 2017;61:42-51.

38. Kim YR, Park B-K, Kim YH, et al. Antidepressant Effect of Fraxinus rhynchophylla Hance Extract in a Mouse Model of Chronic Stress-Induced Depression. Biomed Res Int 2018;2018:8249563.

39. Jin Z, Liang J, Wang J, et al. MCP-induced protein 1 mediates the minocycline-induced neuroprotection against cerebral ischemia/reperfusion injury in vitro and in vivo. J Neuroinflammation 2015;12:39.

40. Zhou P, Xie W, Luo Y, et al. Protective Effects of Total Saponins of Aralia elata (Miq.) on Endothelial Cell Injury Induced by TNF- $\alpha$ via Modulation of the PI3K/Akt and NF-кB Signalling Pathways. Int J Mol Sci 2018;20:36.

41. Zhou P, Xie W, Luo Y, et al. Inhibitory Effects of Ginsenoside Rb1 on Early Atherosclerosis in ApoE/- Mice via Inhibition of Apoptosis and Enhancing Autophagy. Molecules 2018;23:2912.

42. Cai YJ, Wang F, Chen ZX, et al. Hashimoto's thyroiditis induces neuroinflammation and emotional alterations in euthyroid mice. J Neuroinflammation 2018;15:299.

43. Lee B, Sur B, Cho SG, et al. Ginsenoside Rb1 rescues anxiety-like responses in a rat model of post-traumatic stress disorder. J Nat Med 2016;70:133-44.

44. Hui X, Liu Y, Zhang B, et al. The antidepressant effects and mechanism of action of total saponins from the caudexes and leaves of Panax notoginseng in animal models of depression. Phytomedicine 2011;18:731-8.

45. Galeotti N, Bartolini A, Ghelardini C. Blockade of intracellular calcium release induces an antidepressantlike effect in the mouse forced swimming test. Neuropharmacology 2006;50:309-16.

46. Song Q, Fan C, Wang P, et al. Hippocampal CA1 $\beta \mathrm{CaMKII}$ mediates neuroinflammatory responses via COX-2/PGE2 signaling pathways in depression. J Neuroinflammation 2018;15:338.

47. Yu RK, Park BK, Kim YH, et al. Antidepressant Effect ofFraxinus rhynchophyllaHance Extract in a Mouse Model of Chronic Stress-Induced Depression. Biomed Res Int 2018;2018:1-12.

48. Sung U, Binda F, Savchenko V, et al. Ca 2+ dependent surface trafficking of norepinephrine transporters depends on threonine 30 and $\mathrm{Ca} 2+$ calmodulin kinases. J Chem
Neuroanat 2017;83-84:19-35.

49. Huang HJ, Zhu XC, Han QQ, et al. Ghrelin alleviates anxiety- and depression-like behaviors induced by chronic unpredictable mild stress in rodents. Behav Brain Res 2017;326:33-43.

50. Willner P. The chronic mild stress procedure as an animal model of depression: valid, reasonably reliable, and useful. Psychopharmacology 1997;134:371-7.

51. Chiba S, Numakawa T, Ninomiya M, et al. Chronic restraint stress causes anxiety- and depression-like behaviors, downregulates glucocorticoid receptor expression, and attenuates glutamate release induced by brain-derived neurotrophic factor in the prefrontal cortex. Prog Neuropsychopharmacol Biol Psychiatry 2012;39:112-9.

52. Liu X, Zheng X, Du G, et al. Brain metabonomics study of the antidepressant-like effect of Xiaoyaosan on the CUMSdepression rats by (1)H NMR analysis. J Ethnopharmacol 2019;235:141-54.

53. Liang S, Wang T, Hu X, et al. Administration of Lactobacillus helveticus NS8 improves behavioral, cognitive, and biochemical aberrations caused by chronic restraint stress. Neuroscience 2015;310:561-77.

54. Tong J, Zhou Z, Qi W, et al. Antidepressant effect of helicid in chronic unpredictable mild stress model in rats. Int Immunopharmacol 2019;67:13-21.

55. Wang JM, Pei LX, Zhang YY, et al. Ethanol extract of Rehmannia glutinosa exerts antidepressant-like effects on a rat chronic unpredictable mild stress model by involving monoamines and BDNF. Metab Brain Dis 2018;33:885-92.

56. He D, Sai X, Wang N, et al. Camellia euphlebia exerts its antidepressant-like effect via modulation of the hypothalamic-pituitary-adrenal axis and brain monoaminergic systems. Metab Brain Dis 2018;33:301-12.

57. Gupta M, Wiener HL. Effects of deprenyl on monoamine oxidase and neurotransmitters in the brains of MPTPtreated aging mice. Neurochem Res 1995;20:385.

58. Blier P, Montigny CD. Antidepressant Monoamine Oxidase Inhibitors Enhance Serotonin but not Norepinephrine Neurotransmission. Psychopharmacol Ser 1987;3:127.

59. Zhu X, Gao R, Liu Z, et al. Ginsenoside Rg1 reverses stress-induced depression-like behaviors and BDNF expression within the prefrontal cortex. Eur J Neurosci 2016;44:1878-85.

60. Lian $\mathrm{N}$, Niu Q, Lei Y, et al. MiR-221 is involved in depression by regulating $W n t 2 / C R E B / B D N F$ axis in hippocampal neurons. Cell Cycle 2018;17:2745-55.

61. Wang J, Li X, He S, et al. Regulation of the kynurenine 
metabolism pathway by Xiaoyao San and the underlying effect in the hippocampus of the depressed rat. J

Ethnopharmacol 2018;214:13-21.

62. Luo Y, Kuang S, Li H, et al. cAMP/PKA-CREBBDNF signaling pathway in hippocampus mediates cyclooxygenase 2-induced learning/memory deficits of rats subjected to chronic unpredictable mild stress. Oncotarget 2017;8:35558-72.

63. Kandel ER. The molecular biology of memory: cAMP, PKA, CRE, CREB-1, CREB-2, and CPEB. Mol Brain 2012;5:14.

64. Jiang B, Xiong Z, Yang J, et al. Antidepressant-like effects of ginsenoside $\mathrm{Rg} 1$ are due to activation of the BDNF signalling pathway and neurogenesis in the hippocampus. Br J Pharmacol 2012;166:1872-87.

65. Li C, Xu X, Zhang X, et al. Activation of ERK/CREB/ BDNF pathway involved in abnormal behavior of neonatally Borna virus-infected rats. Neuropsychiatr Dis Treat 2018;14:3121-32.

66. Wu X, Tang B, Liao X, et al. Suppressive effects of the supercritical-carbon dioxide fluid extract of Chrysanthemum indicum on chronic unpredictable mild stress-induced depressive-like behavior in mice. Food Funct 2019;10:1212-24.

67. Zhong X, Li G, Qiu F, et al. Paeoniflorin Ameliorates Chronic Stress-Induced Depression-Like Behaviors and Neuronal Damages in Rats via Activation of the ERKCREB Pathway. Front Psychiatry 2019;9:772.

68. Yan T, He B, Wan S, et al. Antidepressant-like effects and cognitive enhancement of Schisandra chinensis in chronic unpredictable mild stress mice and its related mechanism. Sci Rep 2017;7:6903.

69. Zhou SM, Guan SY, Yang L, et al. Cucurbitacin IIa exerts antidepressant-like effects on mice exposed to chronic

Cite this article as: Xie W, Meng X, Zhai Y, Ye T, Zhou P, Nan F, Sun G, Sun X. Antidepressant-like effects of the Guanxin Danshen formula via mediation of the CaMK IICREB-BDNF signalling pathway in chronic unpredictable mild stress-induced depressive rats. Ann Transl Med 2019;7(20):564. doi: 10.21037/atm.2019.09.39 unpredictable mild stress. Neuroreport 2017;28:259-67.

70. Huang C, Yang X, Zeng B, et al. Proteomic analysis of olfactory bulb suggests CACNA1E as a promoter of CREB signaling in microbiota-induced depression. J Proteomics 2019;194:132-47.

71. Wu X, Mcmurray CT. Calmodulin kinase II attenuation of gene transcription by preventing cAMP response elementbinding protein (CREB) dimerization and binding of the CREB-binding protein. J Biol Chem 2001;276:1735-41.

72. Bass M, Pant HC, Gainer H, et al. Calcium/calmodulindependent protein kinase II in squid synaptosomes. J Neurochem 1987;49:1116-23.

73. Walaas SI, Gorelick FS, Greengard P. Presence of calcium/ calmodulin-dependent protein kinase II in nerve terminals of rat brain. Synapse 1989;3:356-62.

74. Zhu A, Shen L, Xu L, et al. Wnt5a mediates chronic postthoracotomy pain by regulating non-canonical pathways, nerve regeneration, and inflammation in rats. Cell Signal 2018;44:51.

75. Herzog DP, Wegener G, Lieb K, et al. Decoding the Mechanism of Action of Rapid-Acting Antidepressant Treatment Strategies: Does Gender Matter? Int J Mol Sci 2019;20:949.

76. Sanwald S, Widenhorn-Muller K, Wernicke J, et al. Depression Is Associated With the Absence of Sex Differences in the 2D:4D Ratio of the Right Hand. Front Psychiatry 2019;10:483.

77. Archer J. The reality and evolutionary significance of human psychological sex differences. Biol Rev Camb Philos Soc 2019;94:1381-415.

78. Eliot L, Richardson SS. Sex in Context: Limitations of Animal Studies for Addressing Human Sex/Gender Neurobehavioral Health Disparities. J Neurosci 2016;36:11823-30. 

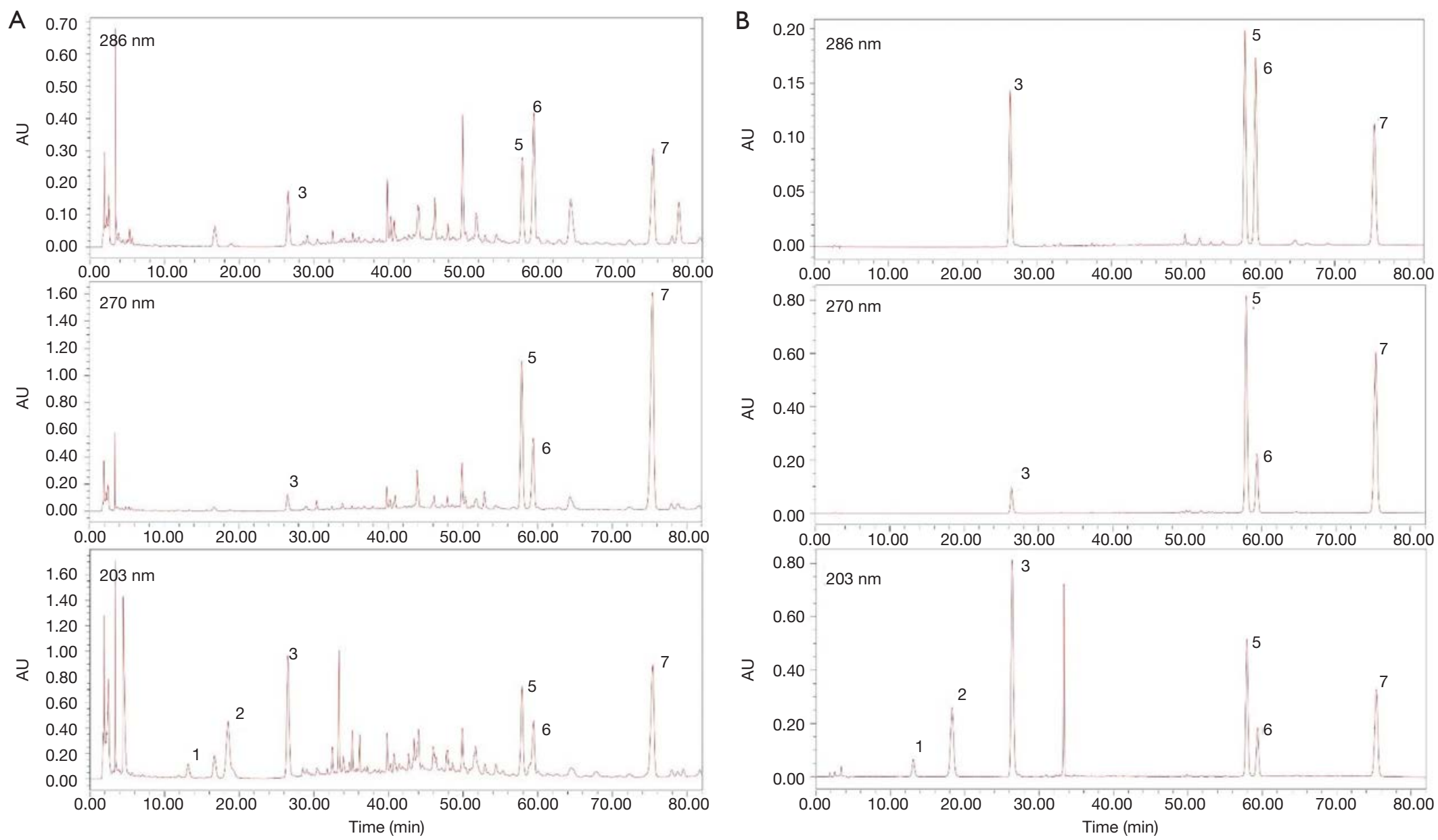

Figure S1 The chromatograms of GXDSF detection via the high performance liquid chromatography. (A) the GXDSF sample chromatograms; (B) the reference substance chromatograms. The detection wavelength was set at $203 \mathrm{~nm}$ (notoginsenoside R1, ginsenoside Rg1 and ginsenoside Rb1), $270 \mathrm{~nm}$ (cryptotanshinone, tanshinone I and tanshinone IIA) and $286 \mathrm{~nm}$ (salvianolic acid B). The percentage of notoginsenoside R1 (No. 1) is about 2.3\%, ginsenoside Rg1 is 9.4\% (No. 2), salvianolic acid B is 0.5\% (No. 3), ginsenoside Rb1 is 8.6\% (No. 4), cryptotanshinone is $0.85 \%$ (No. 5), tanshinone I is $0.55 \%$ (No. 6), tanshinone IIA is 1.7.\% (No. 7). GXDSF, Guanxin Danshen formula. 